\title{
Effect of tangential interface motion on the viscous instability in fluid flow past flexible surfaces
}

\author{
R.M. Thaokar, V. Shankar, and V. Kumaran ${ }^{\mathrm{a}}$ \\ Department of Chemical Engineering, Indian Institute of Science, Bangalore 560 012, India
}

Received 8 November 2000 and Received in final form 20 March 2001

\begin{abstract}
The stability of linear shear flow of a Newtonian fluid past a flexible membrane is analysed in the limit of low Reynolds number as well as in the intermediate Reynolds number regime for two different membrane models. The objective of this paper is to demonstrate the importance of tangential motion in the membrane on the stability characteristics of the shear flow. The first model assumes the wall to be a "spring-backed" plate membrane, and the displacement of the wall is phenomenologically related in a linear manner to the change in the fluid stresses at the wall. In the second model, the membrane is assumed to be a two-dimensional compressible viscoelastic sheet of infinitesimal thickness, in which the constitutive relation for the shear stress contains an elastic part that depends on the local displacement field and a viscous component that depends on the local velocity in the membrane. The stability characteristics of the laminar flow in the limit of low Re are crucially dependent on the tangential motion in the membrane wall. In both cases, the flow is stable in the low Reynolds number limit in the absence of tangential motion in the membrane. However, the presence of tangential motion in the membrane destabilises the shear flow even in the absence of fluid inertia. In this case, the non-dimensional velocity $\left(\Lambda_{t}\right)$ required for unstable fluctuations is proportional to the wavenumber $k\left(\Lambda_{t} \sim k\right)$ in the plate membrane type of wall while it scales as $k^{2}$ in the viscoelastic membrane type of wall $\left(\Lambda_{t} \sim k^{2}\right)$ in the limit $k \rightarrow 0$. The results of the low Reynolds number analysis are extended numerically to the intermediate Reynolds number regime for the case of a viscoelastic membrane. The numerical results show that for a given set of wall parameters, the flow is unstable only in a finite range of Reynolds number, and it is stable in the limit of large Reynolds number.
\end{abstract}

PACS. 83.50.-v Deformation and flow - 47.15.Fe Stability of laminar flows $-47.60 .+\mathrm{i}$ Flows in ducts, channels, nozzles, and conduits

\section{Introduction}

The flow of fluid in channels and tubes with deformable walls is often encountered in biological systems. For instance, in human beings and other mammals, fluids like blood in the cardiovascular system and air in the respiratory system are transported through cylindrical vessels whose walls are soft and deformable. Similar flows are encountered in certain bio-technological applications such as hollow fibre reactors and membrane bioreactors. The dynamics of fluid flow past such "flexible" surfaces is qualitatively different from that of rigid surfaces because of the coupling between the fluid and wall dynamics, and this suggests that the elasticity of the surface could affect the flow. In particular, this coupling could influence the transition from laminar to turbulent flow. In the vicinity of the laminar-turbulent transition, the laminar flow becomes unstable to small perturbations and it is necessary to study the stability characteristics of the flow to predict the parameter values at which there is a transition from stable to unstable modes. There have been various studies,

\footnotetext{
a e-mail: kumaran@chemeng.iisc.ernet.in
}

motivated by drag reduction in marine and aerospace applications [1-5], which have studied the stability problem by modelling the flexible wall to be a thin spring-backed plate membrane. Most of these studies were performed in the high Reynolds number limit, where fluid inertial forces are dominant. There have been other studies on the stability of the laminar flow in flexible tubes and similar systems motivated by biological applications [6-10], which have modelled the wall material as an incompressible viscoelastic solid continuum of finite thickness. The Reynolds number of flows in the biological realm is often very low (typically of $O\left(10^{-2}\right)$ ), and fluid inertia is insignificant. The fluid dynamics in this regime is determined by a balance between viscous stresses in the fluid and elastic stresses in the wall. An interesting possibility of the flow being unstable exists even in the absence of fluid inertia (zero Reynolds number) [6,8]. Kumaran et al. [6] studied the stability of the Couette flow past a viscoelastic medium of finite thickness, and found the flow to be unstable when the velocity of the top plate was greater than a critical value. This instability is qualitatively different from that of flow in rigid-walled channels where fluid inertia plays an important role. 
The nature of hydrodynamic modes of a viscoelastic film of polymeric material at the interface between two Newtonian fluids is a topic of interest in the recent years (see [11] and references therein). In the cases where the interfacial films are made of entangled flexible polymers, insoluble films of entangled polymeric surfactants etc., the dynamical properties of the interface can be adequately modelled by assuming the interfacial film to be a viscoelastic membrane. The assumption of an infinitesimally thin membrane is strictly valid if the amplitude of surface perturbations is large compared to the thickness of the membrane. This criterion is satisfied in biological membranes, where the amplitude of the thermal fluctuations at the surface is large compared to the membrane thickness. Thus, in the limit of a very thin film of viscoelastic material placed between two fluids, it is possible to treat the viscoelastic film as a membrane of infinitesimal thickness and therefore the dynamics of the membrane appear only in the boundary conditions. Harden and Pleiner [11] studied the hydrodynamic modes of such a thin viscoelastic film at the interface between two fluids. They calculated the mode dispersion relations and the dynamic structure factor of thermally induced transverse modes, and found that the coupling between the transverse modes and the in plane longitudinal modes leads to certain unusual features of the surface fluctuations.

Recently, Kumaran and Srivatsan [12] studied the stability of Couette flow past an incompressible membrane, and found that the flow is stable in the limit of zero Reynolds number. They included inertial effects in the fluid using a long wavelength asymptotic analysis and found that inertial stresses destabilise the flow in the long wave limit. Along similar lines, the study of the stability of the developing flow in a flexible-walled channel (modelled as a plate membrane type wall) LaRose and Grotberg [2] showed that the flow is stable in the zero Reynolds number limit; the flow becomes unstable due to inertial stresses at finite Reynolds number. In both these studies, the membrane is assumed to deform only in the normal direction and the tangential motion along the surface is absent. In this paper, the effect of tangential motion at the membrane surface on the stability of the linear shear flow past a flexible membrane is first analysed in the limit of zero Reynolds number. Both the plate membrane model, which has been used in many previous studies, as well as a generalised compressible viscoelastic membrane model, are studied. In the case of plate-membrane type wall models, the majority of previous studies have neglected the tangential motion in the membrane. Most of these studies were, however, restricted to the high Reynolds number limit, and the neglect of the tangential motion is not likely to change their results qualitatively. This is because the tangential stresses in these flows are $O\left(R e^{-1}\right)$ and hence the associated tangential displacements are small, and results are not expected to be altered by tangential wall displacements.

In this paper, we are interested in the low Reynolds number limit and the continuation of these modes to the intermediate Reynolds number regime, and in these situ- ations, the tangential motion in the membrane could be very important. In particular, the presence of tangential motion in the membrane could destabilise the flow even in the limit of zero $R e$. The present analysis shows that in the absence of tangential wall motion, the linear shear flow is always stable in the low Reynolds number limit for the plate-membrane wall model, and this result is in agreement with the previous studies. However, the flow past the plate-membrane wall becomes unstable even in the zero Reynolds number limit, in the presence of tangential motion in the membrane. Two types of tangential damping in the plate-membrane are considered. In the first model (referred to as model A), the damping term is proportional to the velocity of the material points in the plate-membrane. Model A is appropriate when the membrane is connected to a rigid substrate by a spring and viscous dashpot, for example, so that the rate of dissipation of energy due to the dashpot is proportional to the velocity of the surface. Similar models have been used, for example, to model the stability of flow over dolphin skins [4] and also for modelling the flexible walls made of tissue of finite thickness for the respiratory passages [2]. The second model (referred to as model B), the damping term is proportional to the strain rate in the membrane. This mimics, for example, lipid bilayers between two fluids, or a Langmuir-Blodgett film on a liquid surface, which possesses a surface viscosity due to the rate of deformation in the plane of the membrane. However, it should be noted that the normal stress in model $\mathrm{B}$ contains a term proportional to the normal displacement itself, and not to the curvature as in normal bilayers. Therefore, this model mimics a bilayer that is anchored to a substrate, such as the spectrin network in living cells, but where the resistance to tangential motion is due to the in plane strain in the membrane. One of the important results of the analysis is that the stability of flow past the two types of surfaces are very different.

In the case of model $\mathrm{A}$, the fluid velocity $\left(\Lambda_{t}\right)$ required for destabilising the fluctuations scales as the wavenumber $(k)$, i.e. $\Lambda_{t} \sim k$, when the dimensionless dissipation parameter in the wall material $D<D_{\max }$. This implies that for these parameters, long wavelength fluctuations can be rendered unstable by very small fluid velocities. At $D=D_{\max }$, the fluid velocity required for instability reaches a constant value as the wavenumber $k \rightarrow 0$. For $D>D_{\max }$, the flow is always stable to long wavelength fluctuations. For the case of model B with strain rate dependent tangential damping coefficient, the Couette flow is shown to be always unstable in the limit of $k \ll 1$, and $\Lambda_{t} \sim k$ as $k \rightarrow 0$.

The stability of the flow past a compressible viscoelastic membrane at the interface of two fluids is also analysed in this paper. In the case of an incompressible membrane (which is a limiting case of the compressible membrane with the bulk modulus tending to infinity), the tangential deformation is zero for two-dimensional disturbances, and the flow past a membrane of this type is stable at zero Reynolds number [12]. In the opposite limit of a fluid interface, where the bulk and shear moduli of the interface go to zero, the laminar flow is stable at zero Reynolds 
number. The present analysis shows that there exists an instability for a compressible membrane even in the zero Reynolds number limit due to the tangential motion of the membrane. In the low Reynolds number limit the fluid velocity required for destabilising the flow for the compressible membrane scales as $k^{2}$ unlike the $\Lambda_{t} \sim k$ behaviour of the plate-membrane model. These results are then numerically continued to the intermediate Reynolds number regime, and it is found that the instability for compressible membrane is similar to the instability observed for incompressible membrane [12] in the intermediate Reynolds number regime.

In the intermediate Reynolds number regime, the transition Reynolds number $R e_{t}$ scales as $\Sigma^{1 / 2}$ in the entire range of $\Sigma$ for the incompressible membranes [12], where the parameter $\Sigma=\rho \Gamma R /\left(\eta^{a}\right)^{2}$ is only a function of the fluid and membrane parameters and is independent of the fluid velocity. However, for the compressible membrane model analysed in this paper, $R e$ scales as $\Sigma$ in the low $\Sigma$ limit and then crosses over to $R e \propto \Sigma^{1 / 2}$ in the high $\Sigma$ limit and merges with the neutral modes of the incompressible membrane.

It is useful at this point to compare the methods and results of the present work with that of [13-15], where instabilities at relatively high $R e$ in the flow past compliant surfaces are accompanied by marked tangential wall motion. Yeo [13] studied the effect of single and multi-layer compliant walls on the stability of Blasius boundary layer flow over a flexible surface using the parallel flow assumption. The wall medium was modelled as a compressible viscoelastic continuum of finite thickness. The numerical results of [13] revealed three distinct instabilities. In addition to the usual Tollmien-Schlichting instability, there are two other instabilities which are due to the compliance of the wall medium, and these were referred to as $B_{1}$ and $B_{2}$ instabilities. The $B_{1}$ branch was found to exist at high $R e$ (greater than 1000) and high frequencies, while the $B_{2}$ branch was located at $R e$ near 200 and lower frequencies. Increasing the compliance in the wall medium had a destabilising effect on both the $B_{1}$ and $B_{2}$ instabilities. The $B_{2}$ instability, which extends to $R e$ as low as 200, was not continued to $R e \ll 1$, and consequently it is not clear whether the $B_{2}$ branch really extends to the limit of zero Re which is the main focus of this paper. Moreover, Yeo [13] neglected non-parallel effects which become important at low $R e$, and can significantly affect the nature of the $B_{2}$ instability. In contrast, the base Couette flow considered in the present work is an exact parallel shear flow, and the instability reported in this work is not modified by non-parallel effects.

Carpenter and Morris [14] analysed the effect of anisotropic wall compliance on the stability of Blasius boundary layer flow past flexible surface modelled as a spring-backed wall. Their study also invokes the quasiparallel flow assumption and neglects non-parallel effects. Their wall model, like the one used in this paper, incorporates both normal and tangential motion. However, in [14], the normal and tangential displacements are simply related by a constant factor, corresponding to the inclina- tion of the springs. The instabilities identified by them can be broadly classified into three categories: (i) Tollmien Schlichting instability (TSI), (ii) the Travelling wave flutter (TWF) and (iii) the Static Divergence (SD). The TWF and SD exist even in the inviscid limit i.e. in the $R e \rightarrow \infty$ limit, and are essentially inviscid instabilities. However, they are known to exist even in the absence of tangential wall motion [4], and tangential wall motion merely modifies the instability in this case. In the present work, however, the instability is a result of tangential wall motion in the wall medium, and the flow is stable in the absence of tangential wall motion at $R e \ll 1$.

Cooper and Carpenter [15] studied the effect of wall compliance on the stability of rotating-disc boundary layer, where the flexible wall was modelled as compressible viscoelastic continuum of finite thickness. Their primary motivation was to examine whether wall compliance stabilises the inviscid inflection point instabilities existent in boundary layer flow past a rigid rotating disc. Consequently, the instabilities observed in [15] are qualitatively similar to the instabilities in flow past a rigid rotating disc, and the instabilities are modified by wall compliance in a compliant disc. Cooper and Carpenter [15] reported three instabilities: (i) The type 1 instability is an inviscid instability and occurs due to an inflection point in the radial velocity in the boundary layer which gives a Rayleigh type instability in the inviscid limit, (ii) The type 2 instability is a viscous instability and is attributed to Coriolis acceleration, and (iii) The type 3 modes, which are highly damped stable modes, but often undergo "modal coalescence" with type 1 to give a highly unstable absolute instability. Both the type 1 and 2 instabilities have a standing and a travelling mode. All the instabilities reported by Cooper and Carpenter [15] exist even in flow past rigid rotating disc, and are modified by wall compliance. However, the travelling wave type 2 instability exists at lower Re (about 200) and lower frequencies. In this regime, the flow over rigid wall is stable, but there exists an instability in the compliant case. The reason cited in [15] is the large displacements induced at the interface, due to viscous stress terms which arise from the action of Coriolis forces in the system. But even in this case the non parallel effects neglected can become important at low $R e$ and modify the instability. The instability reported in our paper is qualitatively different from that of Cooper and Carpenter [15] as there are no Coriolis forces acting in the system.

\section{Problem formulation}

In this section, the two membrane models are described, and the appropriate governing equations and constitutive relations are provided.

\subsection{Plate model}

The system consists of a fluid of thickness $R$ which is bounded at $z=R$ by a rigid surface moving at a constant velocity $V$, as shown in Figure 1. At the lower boundary 


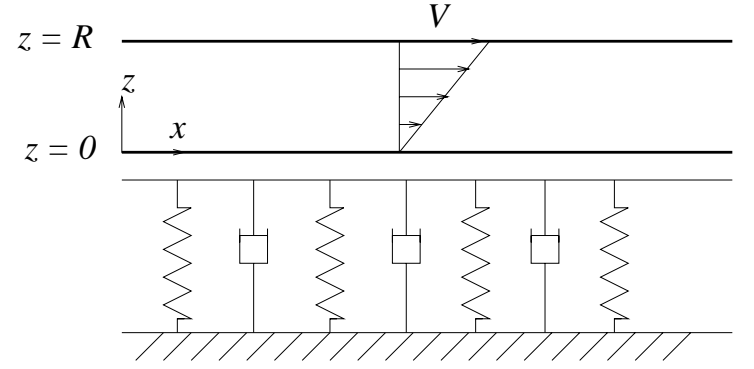

Fig. 1. Configuration for the plate model.

$z=0$, there is an infinitesimal membrane which is modelled as a "plate-membrane" wall. In the plate-membrane wall model $[4,2,1,3,5]$, the flexible wall is modelled as a spring-backed plate which can deform both in the horizontal and vertical directions. The displacement in the horizontal and vertical directions, denoted respectively by $u_{x}^{*}$ and $u_{z}^{*}$, represent the deviation of the material points in the wall material from their equilibrium positions. In the previous studies, a constitutive equation of the following type

$$
\left(D_{n}^{*} \partial_{t}^{*}+B^{*} \partial_{x}^{* 4}-T^{*} \partial_{x}^{* 2}+K^{*}\right) u_{z}^{*}=n_{i} \tau_{i j}^{*} n_{j}
$$

has been used for the normal displacement of the membrane $\left(u_{z}^{*}\right)$, and the tangential displacement $\left(u_{x}^{*}\right)$ is set equal to zero. Here $\tau_{i j}^{*}$ is the total stress tensor in the fluid, and $n_{i}$ is the unit normal to the flexible surface. In the present study, the above condition is augmented by another relation between the tangential stress and the tangential displacement. Two different models are used to describe the tangential motion. In the first model, referred from now on as "model A", the damping term in the tangential direction is proportional to the tangential velocity of material points in the membrane [2].

$$
\left(D_{t}^{*} \partial^{*}{ }_{t}-E^{*} \partial_{x}^{* 2}\right) u_{x}^{*}=t_{i} \tau_{i j}^{*} n_{j} .
$$

In the above equations (1) and (2), $n_{i} \tau_{i j}^{*} n_{j}$ and $t_{i} \tau_{i j}^{*} n_{j}$ are respectively the normal and tangential fluid stress at the interface, and $t_{j}$ is the unit vector tangential to the flexible surface. $D_{n}^{*}$ is the normal wall damping coefficient, $D_{t}^{*}$ is the tangential wall damping coefficient, $B^{*}$ is the flexural rigidity of the plate, $T^{*}$ is the longitudinal tension per unit width, $K^{*}$ is the spring stiffness of the membrane, $E^{*}$ is the bending stiffness of the membrane, $x^{*}$ is the Cartesian coordinate along the wall, $z^{*}$ is the direction normal to the wall, $\partial_{x}^{*} \equiv \partial / \partial x^{*}, t^{*}$ is the dimensional time variable, and $\partial_{t}^{*} \equiv \partial / \partial_{t}^{*}$. Inertial effects are neglected because we are considering the low Reynolds number limit. We have also considered another model, referred to as "model B", where the tangential damping is proportional to the strain rate in the wall

$$
\left(D_{t}^{*} \partial_{x}^{*} \partial_{t}^{*}-E^{*} \partial_{x}^{* 2}\right) u_{x}^{*}=t_{i} \tau_{i j}^{*} n_{j}
$$

It is possible to incorporate an elastic (spring-like) restoring force in the tangential displacement condition (2), but the effect of such a term will be purely stabilising, since the tangential restoring force will tend to decrease tangential displacement in the plate-membrane. The equations describing the wall dynamics are nondimensionalised in the following manner. Lengths are scaled by $R$ the thickness of the fluid layer flowing above the membrane, velocities by $\left(K^{*} R^{2} / \eta\right)$, where $\eta$ is the viscosity of the Newtonian fluid flowing above the membrane wall, $t^{*}$ is scaled by $\eta /\left(K^{*} R\right)$, and the fluid stresses are scaled by $K^{*} R$. The nondimensional governing equations describing the wall dynamics take the following form,

$$
\begin{gathered}
n_{i} \tau_{i j} n_{j}=-T \partial_{x}^{2} u_{z}+D_{n} \partial_{t} u_{z}+B \partial_{x}^{4} u_{z}+u_{z} \\
t_{i} \tau_{i j} n_{j}=-E \partial_{x}^{2} u_{x}+D_{t} \partial_{t} u_{x}
\end{gathered}
$$

for model A, where the nondimensional wall parameters are $T=T^{*} /\left(k^{*} R^{2}\right), D_{n}=D_{n}^{*} R / \eta, D_{t}=D_{t}^{*} R / \eta$, $B=B^{*} /\left(K^{*} R^{4}\right)$ and $E=E^{*} /\left(K^{*} R^{2}\right)$. For model $\mathrm{B}$, the nondimensional governing equation for the tangential displacement is given by

$$
t_{i} \tau_{i j} n_{j}=-E \partial_{x}^{2} u_{x}+D_{t} \partial_{x} \partial_{t} u_{x}
$$

In (4), the imposed pre-tension in the membrane $T$ is assumed to be a $x$-independent constant quantity. It should be noted that the mean shear stress exerted by the Couette flow on the pre-tensioned membrane will cause a flow induced linear variation of wall tension in the membrane. Thus, the total tension is given by $T_{\text {tot }}=T+T_{\text {flow }}$, where $T$ is the imposed ( $x$-independent) pre-tension in the plate membrane, and $T_{\text {flow }}$ is the flow induced tension. In the present analysis, the pre-tension in the membrane $T$ is assumed to be much larger than the flow-induced tension $T_{\text {flow }}$, so that it is possible to neglect $T_{\text {flow }}$ in (4). As shown in Section 3.1, an important feature of the present instability is that it is a low wavenumber (long wavelength) instability, and the $\Lambda$ required for unstable modes is shown to scale as $\Lambda \sim k$ as $k \rightarrow 0$. The flow induced tension in the membrane scales as $\Lambda x$. Since the effective axial length scale for perturbations goes as $k^{-1}$ the tension $T_{\text {flow }}$ scales as $O(1)$. Thus the contribution due to flow induced tension can be neglected in the normal stress balance if $T \gg 1$, and for the results presented in Section 3.1, the values of $T$ chosen are such that this condition is satisfied.

In the following analysis, we set $D_{t}=D_{n}=D$ for simplicity and in order to reduce the parameter space to be probed.

The boundary conditions at the interface between the fluid and the wall are the continuity of velocities and stresses in the normal and tangential directions. At the upper rigid plate $(z=1)$, no-slip boundary conditions are appropriate for both the components of the fluid velocity. The velocity continuity conditions at the membrane surface are

$$
\begin{aligned}
& v_{x}=\partial_{t} u_{x} \\
& v_{z}=\partial_{t} u_{z} .
\end{aligned}
$$

These boundary conditions are supplemented by the stress balance conditions at the interface (4) and (5). 


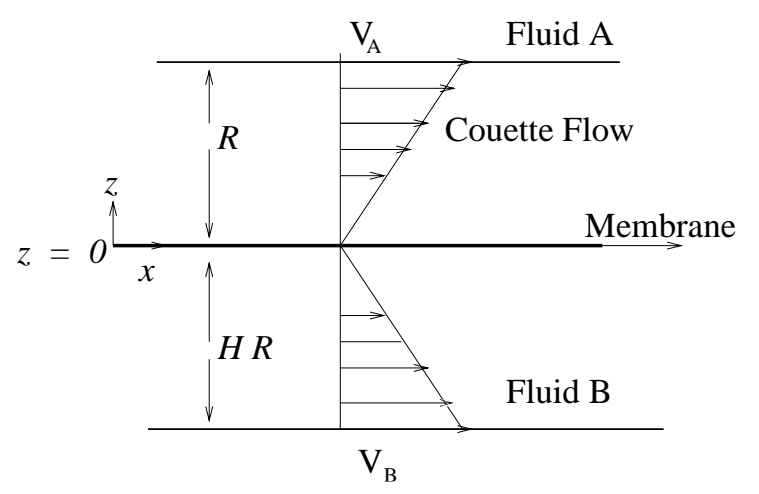

Fig. 2. Configuration for the membrane model.

\subsection{Compressible viscoelastic membrane model}

The system, shown in Figure 2, consists of a compressible viscoelastic membrane of infinitesimal thickness, surface tension $\Gamma^{*}$, bulk modulus $K^{*}$, shear modulus $G^{*}$ and negligible inertia stretched along the interface $z^{*}=0$ between two fluids $\mathrm{A}$ and $\mathrm{B}$ of thickness $R$ and $H R$ respectively. The membrane parameters $K^{*}, \Gamma^{*}$ and $G^{*}$ are defined in units of Force/length. For simplicity, the densities of the two fluids are set to be equal but the viscosities of the two fluids are considered to be different. The surface bounding the fluid A at $z^{*}=R$ moves with a velocity $V_{a}$, while the surface bounding the fluid $\mathrm{B}$ at $z^{*}=-H R$ moves with a velocity $V_{b}$ so that the dimensional velocity profiles are given by $v_{x}^{* a}=\frac{V_{a} z}{R}$ and $v_{x}^{* b}=-\frac{V_{b} z}{R H}$. The lengths in the problem are scaled by the thickness $R$ of the top fluid, the velocities by $\left(\Gamma^{*} / \eta^{a}\right)$ and the time coordinate by $\left(R \eta^{a} / \Gamma^{*}\right)$. Here $\eta^{a}$ is the viscosity of the top fluid layer. The fluid stresses and pressure are scaled by $\left(\Gamma^{*} / R\right)$, while the membrane stresses are scaled by $\left(\Gamma^{*}\right)$. The parameters $\Lambda_{a}=\left(V_{a} \eta^{a} / \Gamma^{*}\right)$ and $\Lambda_{b}=-\left(V_{b} \eta^{a} / \Gamma^{*} H\right)$ are defined so that the scaled mean velocities are

$$
v_{x}^{n}=\Lambda_{n} z
$$

where $n$ is a for fluid A and $b$ for fluid B. The constitutive relation for the membrane stress in case of a $2 \mathrm{D}$ membrane of infinitesimal thickness can be given by [11],

$$
\sigma_{\alpha \beta}^{*}=K^{*} \partial_{\gamma}^{*} u_{\gamma}^{*} \delta_{\alpha \beta}+\left(G^{*}+\eta_{\mathrm{m}}^{*} \partial_{t}^{*}\right)\left(\partial_{\alpha}^{*} u_{\beta}^{*}+\partial_{\beta}^{*} u_{\alpha}^{*}\right) .
$$

Using the scales used above, the non-dimensional form of the above equation becomes

$$
\sigma_{\alpha \beta}=K \partial_{\gamma} u_{\gamma} \delta_{\alpha \beta}+\left(G+\eta_{\mathrm{m}} \partial_{t}\right)\left(\partial_{\alpha} u_{\beta}+\partial_{\beta} u_{\alpha}\right) .
$$

Here, $\sigma_{\alpha \beta}$ is the stress (force per unit length) along the membrane surface, and $u_{\alpha}$ is the displacement vector, along the membrane surface, of material points from their equilibrium positions due to stresses exerted on the membrane. Note that Greek indices are used to denote two dimensional vectors along the membrane surface. In (11), $K=K^{*} / \Gamma^{*}$ is the non dimensionless bulk modulus of the membrane, $G=G^{*} / \Gamma^{*}$ is the non dimensionless shear modulus, $\eta_{\mathrm{m}}=\eta_{\mathrm{m}}^{*} /\left(R \eta^{a}\right)$ is the nondimensional membrane viscosity. The incompressible viscoelastic constitutive relation used in Kumaran and Srivatsan [12, ] is a limiting case of the above general compressible equation with the bulk modulus going to infinity. For two dimensional disturbances, there is a membrane displacement only in one direction along the surface of the membrane (the $x$ direction in 2), and the equation for the stress is

$$
\sigma_{x x}=\left(K+2 G+2 \eta_{\mathrm{m}} \partial_{t}\right) \partial_{x} u_{x} .
$$

For an incompressible membrane, the bulk modulus $K$ goes to infinity, and the displacement in the direction tangential to the membrane is zero, since any finite displacement would cause an infinite stress in the tangential direction. The continuity of tangential velocity condition requires that the tangential velocity in the fluid should also be zero in the linear approximation.

For a compressible viscoelastic membrane, the force balance condition relating the fluid stresses to the tangential stress exerted along the membrane surface is derived in Appendix A. As shown in the appendix, the generalised force balance equation for a $2 \mathrm{D}$ membrane with infinitesimal thickness can be derived as a limiting case of that of a finite thickness gel, in the limit of thickness going to zero. Substituting the membrane constitutive relation in the force balance (69), we obtain the following expression for two dimensional disturbances, where $x$ is the flow direction, and $z$ is the direction normal to the unperturbed membrane surface:

$$
\left[t_{i} \tau_{i j}^{*} n_{j}\right]^{a}-\left[t_{i} \tau_{i j}^{*} n_{j}\right]^{b}+\mathbf{t} \cdot \nabla_{\mathbf{s}}^{*} \cdot \boldsymbol{\sigma}^{*}=\mathbf{0}
$$

where $\nabla_{s}$ is the surface gradient along the membrane surface, and $\boldsymbol{\sigma}^{*}$ is the stress tensor of the membrane (10). The expression for surface gradient $\nabla_{s}$ is given by $\nabla_{s}^{*}=$ $\left[\nabla^{*}-\mathbf{n}\left(\mathbf{n} . \nabla^{*}\right)\right]$, where $\nabla^{*}$ is the three dimensional gradient operator and $\mathbf{n}$ and $\mathbf{t}$ is the unit normal and the unit tangent vector to the membrane surface respectively.

At the upper rigid plate $(z=1)$, no-slip boundary conditions are appropriate for all the components of the fluid velocity. Similarly at the lower rigid plate $(z=-H)$, no slip boundary conditions are to be satisfied for the second fluid. The boundary conditions at the interface between the fluid and the membrane are the continuity of velocities and force balance in the tangential and normal directions. This is different from the incompressible membrane model where the tangential stress balance gets decoupled due to zero tangential velocity condition at the interface. The scaled tangential stress balance condition in the present case is given by (13). The dimensional normal stress balance condition is considered to be of simple form

$$
\left[n_{i} \tau_{i j}^{*} n_{j}\right]^{a}-\left[n_{i} \tau_{i j}^{*} n_{j}\right]^{b}=-\Gamma^{*} \nabla_{s}^{*} \cdot \mathbf{n}
$$

where the last term on the right side of above equation is due to surface tension and bending rigidity is considered negligible. This when non-dimensionalised becomes

$$
\left[n_{i} \tau_{i j} n_{j}\right]^{a}-\left[n_{i} \tau_{i j} n_{j}\right]^{b}=-\nabla_{s} \cdot \mathbf{n} .
$$

The surface tension term in the normal stress balance contains three parts: $\Gamma^{*}=\Gamma_{\text {int }}^{*}+\Gamma_{\text {imp }}^{*}+\Gamma_{\text {flow }}^{*}$. Here $\Gamma_{\text {int }}^{*}$ is the intrinsic surface tension of the membrane fluid interface, and arises due to the difference in the surface tension 
between the viscoelastic membrane and the two fluids on either side. $\Gamma_{\mathrm{imp}}^{*}$ is the imposed $x$-independent tension in the membrane in the absence of flow. $\Gamma_{\text {flow }}^{*}$ is the tension due to tangential stresses exerted by the mean flow of the adjacent fluids on the membrane. This flow induced $x$-dependent tension in the membrane is given by the expression $\Gamma_{\text {flow }}^{*} / \Gamma^{*}=\Lambda x$. It is shown in Section 3.3 that the transition velocity $\Lambda_{t}$ for the unstable modes scales as $k^{2}$. Since the axial effective length scale of perturbations goes as $k^{-1}$ the flow induced tension scales as $O(k)$. Therefore, in the following analysis, we neglect the flow induced tension in the $k \rightarrow 0$ limit, and retain only the $O(1)$ intrinsic and imposed surface tension in the normal stress balance.

The velocity continuity conditions at the interface are

$$
\begin{aligned}
& v_{z}^{a}=v_{z}^{b}=\partial_{t} u_{z} \\
& v_{x}^{a}=v_{x}^{b}=\partial_{t} u_{x}
\end{aligned}
$$

where $u_{x}$ couples the stresses in the fluid and the membrane (13).

The equations governing the incompressible Newtonian fluid dynamics for fluids $\mathrm{A}$ and $\mathrm{B}$ are the usual Navier-Stokes mass and momentum equations. The nondimensional governing equations for the fluid, after using the above scales for nondimensionalisation, are given by

$$
\begin{gathered}
\partial_{i} v_{i}^{n}=0 \\
\frac{R e}{\Lambda_{a}}\left(\partial_{t}+v_{j}^{n} \partial_{j}\right) v_{i}^{n}=-\partial_{i} p^{n}+\frac{\eta^{n}}{\eta^{a}} \partial_{j}^{2} v_{i}^{n}
\end{gathered}
$$

where the Reynolds number $R e$ is defined as $R e=$ $\left(\rho V_{a} R / \eta^{a}\right)$, and $\eta^{r}=\eta^{b} / \eta^{a}$ is the relative fluid viscosity. The scaled stress tensor in the fluid is given by

$$
\tau_{i j}^{n}=-p^{n} \delta_{i j}+\frac{\eta^{n}}{\eta^{a}}\left(\partial_{i} v_{j}^{n}+\partial_{j} v_{i}^{n}\right) .
$$

In the above expressions $n$ is $a$ for fluid A and $b$ for fluid B.

In the limit of $R e \rightarrow 0$, the inertial terms in the momentum balance of the fluid can be neglected when compared to the viscous and pressure gradient terms, and the governing equations for the fluid reduce to

$$
-\partial_{i} p^{n}+\frac{\eta^{n}}{\eta^{a}} \partial_{j}^{2} v_{i}^{n}=0 .
$$

\section{Stability analysis}

\subsection{Plate model}

The perturbation to the normal and tangential displacements $u_{z}$ and $u_{x}$ in the membrane model are expressed in the form of Fourier modes:

$$
u_{z}=\tilde{u}_{z}(z) \mathrm{e}^{\mathrm{i} k x+s t}, u_{x}=\tilde{u}_{x}(z) \mathrm{e}^{\mathrm{i} k x+s t} .
$$

Here, $k$ is the wavenumber, $s$ is the growth rate of the perturbations, $\tilde{u}_{z}$ and $\tilde{u}_{x}$ are respectively the Fourier components of the displacements $u_{z}$ and $u_{x}$. The growth rate $s$ is in general a complex quantity, and the flow is temporally unstable if the real part of $s$ is positive. The boundary conditions $(7,8,4,5)$ at the interface between the fluid and the wall have to be applied at the perturbed position of the interface. However, in the linear stability analysis, the velocity and stress fields due to the mean flow and perturbations at the perturbed interface are expanded in a Taylor series about their values at the unperturbed interface at $z=0$. Only the linear terms in the series expansion are retained and higher-order terms are neglected to obtain the following boundary conditions in which all quantities are evaluated at the unperturbed interface.

$$
\tilde{v}_{z}=s \tilde{u}_{z}, \quad \tilde{v}_{x}+\Lambda \tilde{u}_{z}=s \tilde{u}_{x}
$$

In this equation, $\tilde{v}_{z}$ and $\tilde{v}_{x}$ are the Fourier components of the fluid, $x$ and $z$ directional disturbance velocities. The fluid normal and tangential stresses at the perturbed interface are respectively given by $n_{i} \tau_{i j}^{\text {tot }} n_{j}$ and $t_{i} \tau_{i j}^{\text {tot }} n_{j}$. Here, $n_{j}$ and $t_{j}$ are respectively the unit vectors in the normal and tangential directions to the perturbed interface, $\tau_{i j}^{\text {tot }}$ is total fluid stress tensor at the interface which is given by the sum of the mean and perturbation stresses, i.e. $\tau_{i j}^{\text {tot }}=\tau_{i j}^{\text {mean }}+\tau_{i j}^{\prime}$, where $\tau_{i j}^{\prime}$ is the stress tensor due to perturbations. The expressions for the unit vectors normal and tangential to the deformed surface, to linear order in the perturbation quantities, are:

$$
\mathbf{n}=\mathbf{e}_{z}-\partial_{x} u_{z} \mathbf{e}_{x}, \mathbf{t}=\mathbf{e}_{x}+\partial_{x} u_{z} \mathbf{e}_{z} .
$$

Here $\mathbf{e}_{x}$ and $\mathbf{e}_{z}$ are unit vectors in the horizontal $x$-and vertical $z$-directions respectively. The mean shear stress tensor at the interface is $\Lambda \mathbf{e}_{x} \mathbf{e}_{z}$. Therefore, the normal stress at the perturbed interface $n_{i} \tau_{i j}^{\text {tot }} n_{j}$ becomes, to linear order in perturbation quantities:

$$
\mathbf{n} \cdot \boldsymbol{\tau} \cdot \mathbf{n}=-2 \Lambda \partial_{x} u_{z}-p_{f}+2 \partial_{z} v_{z}
$$

The term $-2 \Lambda \partial_{x} u_{z}$ represents the mean stress at the interface due to the variation in the unit normal of the perturbed interface. Therefore, the normal stress boundary condition at the unperturbed interface $(z=0)$ is given, to linear order, by

$$
\begin{aligned}
&-2 \Lambda \mathrm{i} k \tilde{u}_{z}+\left(-\tilde{p}+2 \partial_{z} \tilde{v}_{z}\right)= \\
&\left(1+D s+T k^{2}+B k^{4}\right) \tilde{u}_{z} .
\end{aligned}
$$

The tangential stress boundary condition at the unperturbed interface $z=0$ is given, for model A:

$$
\left(\partial_{z} \tilde{v}_{x}+\mathrm{i} k \tilde{v}_{z}\right)=\left(E k^{2}+D s\right) \tilde{u}_{x}
$$

and for model B:

$$
\left(\partial_{z} \tilde{v}_{x}+\mathrm{i} k \tilde{v}_{z}\right)=\left(E k^{2}+\mathrm{i} k D s\right) \tilde{u}_{x} .
$$

The tangential stress boundary condition does not have any contributions from the variation of unit normal in the 
interface, since these contributions are non-linear in the perturbation quantities. For the case of membrane walls without tangential motion, we set $\tilde{v}_{x}=0$ at $z=0$ and the tangential stress condition is omitted.

The governing equations in the zero $R e$ limit are the incompressible Stokes equations which, in terms of the non-dimensional variables, are

$$
\partial_{i} v_{i}=0,-\partial_{i} p+\partial_{j}^{2} v_{i}=0
$$

The pressure and velocities in the above equation are scaled as given in Section 2.1. The dynamical quantities are perturbed by disturbances of the form

$$
v_{i}(x, z, t)=\tilde{v}_{i}(z) \exp [\mathrm{i} k x+s t] .
$$

The governing equations in the fluid, when expressed in terms of the Fourier modes, are

$$
\begin{array}{r}
\partial_{z} \tilde{v}_{z}+\mathrm{i} k \tilde{v}_{x}=0 \\
-\mathrm{i} k \tilde{p}+\left(\partial_{z}^{2}-k^{2}\right) \tilde{v}_{x}=0 \\
-\partial_{z} \tilde{p}+\left(\partial_{z}^{2}-k^{2}\right) \tilde{v}_{z}=0 .
\end{array}
$$

The eigenfunctions for the fluid velocity field can easily be obtained from the above governing stability equations:

$$
\tilde{v}_{z}(z)=A_{1} \mathrm{e}^{k z}+A_{2} z \mathrm{e}^{k z}+A_{3} \mathrm{e}^{-k z}+A_{4} z \mathrm{e}^{-k z} .
$$

There are four undetermined constants in the fluid velocity field. These are determined from the four boundary conditions, two conditions for the fluid velocity at $z=1$, and two continuity conditions for the fluid velocity at $z=0$. In the two velocity continuity conditions at the interface, $\tilde{u}_{x}$ and $\tilde{u}_{z}$ are related by the membrane "constitutive" relations (4 and 5). This gives rise to a set of four homogeneous equations for the coefficients $A_{1}, A_{2}, A_{3}$ and $A_{4}$. The determinant of the $4 \times 4$ coefficient matrix is set equal to zero in order to obtain the characteristic equation for $s$. The characteristic equation is a quadratic equation in the growth rate $s$, and can be solved to obtain the growth rate as a function of the wave number and wall parameters. The results for the case without tangential motion is discussed first. It turns out that, in all the cases studied, the real part of the growth rate is always negative indicating that the flow is stable. Increasing the flow velocity $\Lambda$ merely alters the frequency of the perturbations. Thus the flow is stable in the limit of zero $R e$ in the absence of tangential wall motion in the plate membrane.

In the presence of tangential motion in the wall, it is shown below that the flow could be unstable even in the zero Reynolds number limit. The analytical expression for the growth rate is rather complicated, and in order to examine the effect of various terms such as $E, T, B$ etc., it is useful to carry out a low wavenumber expansion. It also turns out that the modes with $k \rightarrow 0$ are the most unstable modes, and the low $k$ asymptotic analysis clearly brings out the effect of the relevant physical parameters for the most unstable modes. In the limit of $k \rightarrow 0$, the continuity equation (31) indicates that $\tilde{v}_{z} \sim O(k) \tilde{v}_{x}$, and the $x$-momentum equation (32) shows that $\tilde{p_{f}} \sim k^{-1} \tilde{v}_{x}$. Therefore, it is appropriate to expand the quantities in an asymptotic series in the small parameter $k$ in the following fashion:

$$
\begin{aligned}
\tilde{v}_{x}=\left(\tilde{v}_{x}^{0}+k \tilde{v}_{x}^{1}+\cdots\right), & \tilde{v}_{z}=k\left(\tilde{v}_{z}^{0}+k \tilde{v}_{z}^{1}+\cdots\right), \\
\tilde{p_{f}} & =k^{-1}\left(\tilde{p_{f}}{ }^{0}+k{\tilde{p_{f}}}^{1}+\cdots\right) .
\end{aligned}
$$

We first discuss the low $k$ analysis for model A. The normal stress balance (26) indicates that $\tilde{u}_{z}$ is $O\left(k^{-1}\right)$, since $\tilde{p_{f}}$ is $O\left(k^{-1}\right)$. Therefore, it is appropriate to expand

$$
\tilde{u}_{z}=k^{-1}\left(\tilde{u}_{z}^{0}+k \tilde{u}_{z}^{1}+\cdots\right) .
$$

From the normal velocity continuity condition (23), it can then be inferred that $s$ is $O\left(k^{2}\right)$, and thus it is appropriate to expand

$$
s=k^{2}\left(s^{0}+k s^{1}+\cdots\right) .
$$

For model A, the tangential stress condition (27) then shows that $\tilde{u}_{x}$ is $O\left(k^{-2}\right)$, since $\tilde{v}_{x}$ is an $O(1)$ quantity, and $E$ is assumed to be an $O(1)$ quantity. So, $\tilde{u}_{x}$ is expanded as

$$
\tilde{u}_{x}=k^{-2}\left(\tilde{u}_{x}^{0}+\cdots\right)
$$

From the tangential velocity continuity condition (23) it can then be inferred that $\Lambda$ is $O(k)$ in order for a balance to be achieved between the three terms in the boundary condition. It is convenient to set $\Lambda=k \Lambda_{0}$, where $\Lambda_{0}$ is an $O(1)$ quantity. Substituting all these expansions in the governing equations,

$$
\partial_{z} \tilde{v}_{z}^{0}+\mathrm{i} \tilde{v}_{x}^{0}=0,-\mathrm{i} \tilde{p}_{f}^{0}+\partial_{z}^{2} \tilde{v}_{x}^{0}=0, \partial_{z} \tilde{p}_{f}^{0}=0 .
$$

The above set of governing equations can be easily solved to give the following expression for $\tilde{v}_{z}^{0}$ :

$$
\tilde{v}_{z}^{0}=A_{1}+A_{2} z+A_{3} z^{2}+A_{4} z^{3} .
$$

Two of the constants (say $A_{1}$ and $A_{2}$ ) can be eliminated by using the boundary conditions at $z=1$, and the two remaining constants have to be determined from the continuity conditions at $z=0$. The continuity conditions, to leading order in $k$ are given by

$$
\begin{aligned}
\tilde{v}_{z}^{0} & =s^{0} \tilde{u}_{z}^{0}, \tilde{v}_{x}^{0}+\Lambda^{0} \tilde{u}_{z}^{0}=s^{0} \tilde{u}_{x}^{0} \\
-\tilde{p}_{0} & =\tilde{u}_{z}^{0}, \partial_{z} \tilde{v}_{x}^{0}=\left(E+s^{0} D\right) \tilde{u}_{x}^{0} .
\end{aligned}
$$

From the low $k$ expansion of the boundary conditions, the following points can be observed. In the normal stress balance (compare 26 and 42), the tension term, the bending term and the dissipation term are small compared to the spring term in the low $k$ limit. The damping term appears only in the tangential stress condition. Similarly, the term $-2 \mathrm{i} k \Lambda \tilde{u}_{z}$ in $(26)$ due to the variation of the unit normal in the perturbed interface, is small compared to the other terms in the low $k$ limit. The quantities $\tilde{u}_{x}^{0}$ and $\tilde{u}_{z}^{0}$ can be eliminated from (42) and these can be substituted in (41), 


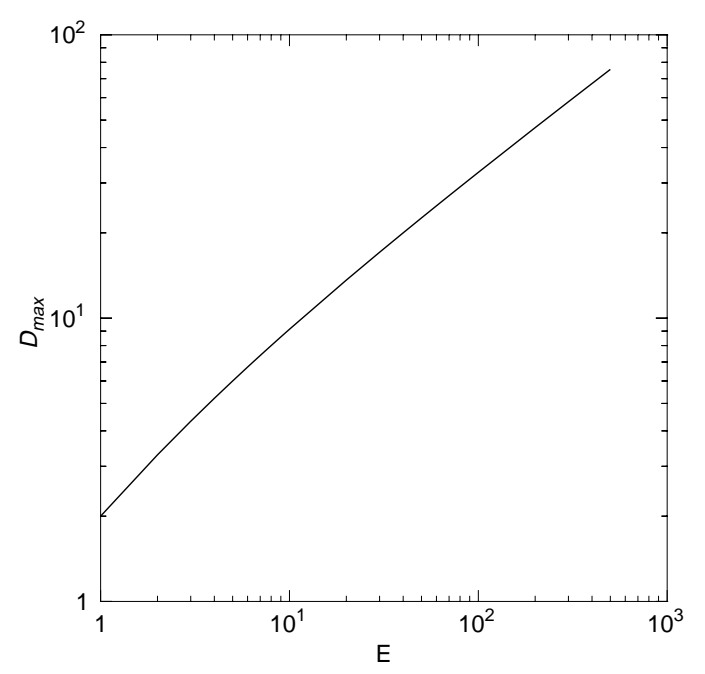

Fig. 3. Variation of $D_{\max }$ with $E$ as predicted by the low $k$ asymptotic analysis for model A with velocity dependent tangential damping in the plate membrane.

to yield a set of homogeneous equations for the two undetermined constants $A_{3}$ and $A_{4}$. In order to have non-trivial solutions to this system of equations, the determinant of this system is set equal to zero, and this yields a characteristic equation for $s^{0}$. Note here that $s^{0}$ is a complex quantity, and if the real part of $s^{0}$ is positive, then the flow is unstable. In order to determine the neutral modes (i.e. $\operatorname{Re}\left[s^{0}\right]=0$ ), the real part of $s^{0}$ is set equal to zero in the characteristic equation, and this gives an expression for $\Lambda_{0}$ in terms of the other parameters $E$ and $D$. The $\Lambda_{0}$ required for neutrally stable modes then turns out to be

$$
\Lambda_{0}=\frac{(4+D+12 E)^{1 / 2}}{\left(-36 D+\frac{432 E}{4+D+12 E}+\frac{432 D E}{4+D+12 E}\right)^{1 / 2}} .
$$

It is first instructive to analyse the behaviour of the neutrally stable modes for $D=0$, and in this case the above expression becomes

$$
\Lambda_{0}=\frac{(1+3 E)}{3^{3 / 2} E^{1 / 2}}
$$

Therefore, the low $k$ asymptotic analysis shows that if $\Lambda \equiv$ $k \Lambda_{0}>k(1+3 E) /\left(3^{3 / 2} E^{1 / 2}\right)$, the flow becomes unstable in the limit of $k \rightarrow 0$, and all the parameters such as $T, B$ and the normal damping coefficient are irrelevant to the low $k$ instability.

For nonzero $D$ in (43), it turns out that for a given $E$, there exists a maximum value of $D \equiv D_{\max }$ below which the low $k$ instability exists, for $D>D_{\max } \Lambda_{0}$ diverges, and the low $k$ instability disappears. Figure 3 shows the variation of $D_{\max }$ with $E$, and this indicates that $D_{\max }$ increases proportional to $E^{1 / 2}$ for large values of $E$. All the results discussed above are for the case of model $A$, where the tangential damping is proportional to the tangential velocity of the material points (see $(27)$ ).

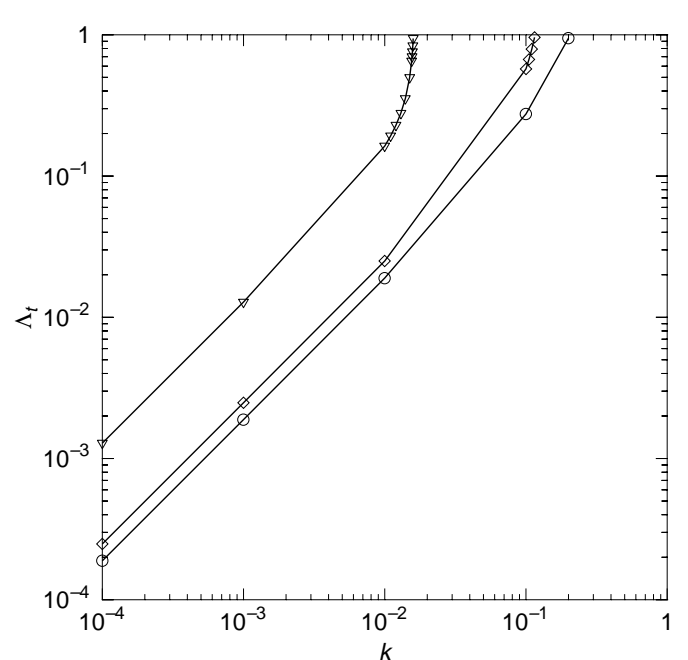

Fig. 4. Variation of transition velocity $\Lambda_{t}$ with $k$ for model A, for different values of the dimensionless dissipation parameter: (॰) $D=0,(\diamond) D=5,(\nabla) D=9$ with $E=10$ and $T=100$, $B=10^{-2}$.

We now present some numerical results for neutral modes in the limit of zero $R e$ for model A, where the restriction of low $k$ is removed, and neutral stability curves are presented for finite wavenumber modes. These results are obtained by setting the real part of the growth rate to be zero in the analytical solution for the growth rate, and the imaginary part of the growth rate and $\Lambda$ were determined numerically using a Newton-Raphson method. In Figure 4, the variation of $\Lambda_{t}$ (the base flow velocity above which flow becomes unstable) with $k$ is shown, and this plot indicates that $\Lambda_{t}$ scales as $k$ for low $k$. This plot indicates that as $D$ is increased, the unstable region in the $\Lambda_{t}-k$ plane shrinks, indicating that the damping in the wall plays a purely stabilising role. This figure also shows that for $k \sim 1$, the $\Lambda_{t}$ required for unstable modes starts diverging, indicating that the tension term is again purely stabilising. In Figure 5 the variation of $\Lambda_{t}$ with $k$ is plotted for two different values of $T$, for fixed values of the remaining parameters. This figure illustrates that as the tension $T$ is increased, the $\Lambda_{t}$ required for producing short wavelength (high $k$ ) fluctuations increases, again indicating the stabilising action of the wall tension $T$.

The behaviour of unstable modes for the case of model B (see (28)), where the tangential damping term is proportional to the strain rate, is discussed next. There is only one modification needed in the asymptotic analysis for model B, and this change is related to the tangential stress condition. In the tangential stress condition for model B (28), the dissipation term is $O(k)$ smaller than the remaining terms, and hence it does not appear to leading order in $k$. All the other boundary conditions and scalings remain the same as in model A. The leading order tangential stress condition, therefore takes the following form:

$$
\partial_{z} \tilde{v}_{x}^{0}=E \tilde{u}_{x}^{0}
$$

Using this and the other three boundary conditions from model $\mathrm{A}$, the $\Lambda_{0}$ required for unstable modes for model $\mathrm{B}$ 


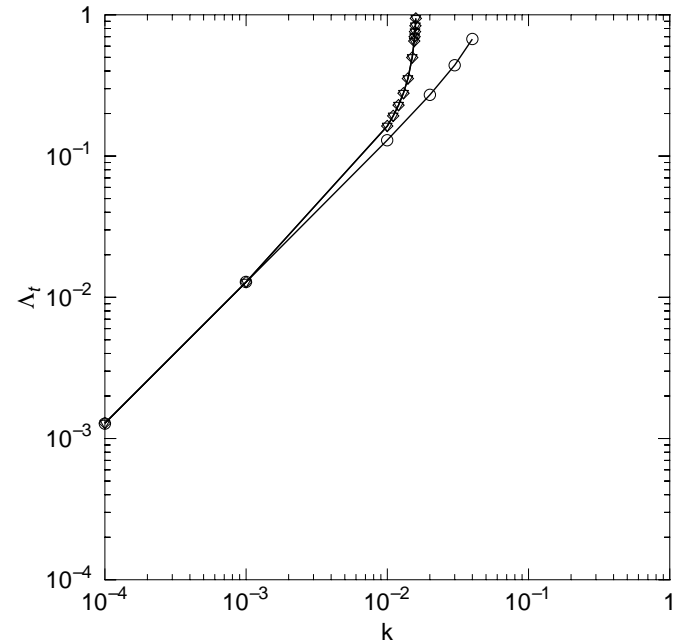

Fig. 5. Variation of transition velocity $\Lambda_{t}$ with $k$ for model A, for different values of $T((\diamond) T=100,(\circ) T=10)$ for fixed $D=9$ and $E=10, B=10^{-2}$.

is then found to be identical to that of model A with $D=0$ (44). Therefore, in model B, the tangential damping term does not have an effect on the low wavenumber instability in the limit $k \rightarrow 0$, and the flow is unstable in the low $k$ limit if $\Lambda>k(1+3 E) /\left(3^{3 / 2} E^{1 / 2}\right)$.

In Figure 6 , the variation of $\Lambda_{t}$ with $k$ is plotted for model $\mathrm{B}$, for different values of wall damping parameter $D$. For small $k, \Lambda_{t}$ scales as $k$, in agreement with the low $k$ analysis. This figure also clearly indicates that, as predicted by the asymptotic analysis, wall damping $D$ indeed does not affect the low wavenumber instability. The effect of damping is to increase the $\Lambda_{t}$ at $k \sim 1$, and this again indicates that the wall damping plays a purely stabilising role in this instability.

\subsection{Viscoelastic membranes}

As in the case of the plate model, normal mode perturbations are imposed on the fluid velocity field and the membrane displacement fields. Only two-dimensional perturbations are considered here because it can be shown (Appendix B) that two-dimensional disturbances are always more unstable than three dimensional ones in the zero Reynolds number limit (Squire's theorem) [16]. Substituting the perturbation variables into the linearised governing equations for the fluid and the membrane, the following linearised equations are obtained:

$$
\partial_{z} \tilde{v}_{z}^{n}+\mathrm{i} k \tilde{v}_{x}^{n}=0
$$

$$
\begin{gathered}
-\mathrm{i} k \tilde{p}^{n}+\left[-\frac{R e}{\Lambda_{a}}\left(s+\Lambda_{n} \mathrm{i} k z\right)+\frac{\eta^{n}}{\eta^{a}}\left(\partial_{z}^{2}-k^{2}\right)\right] \tilde{v}_{x}^{n} \\
-\frac{R e \Lambda_{n}}{\Lambda_{a}} \tilde{v}_{z}^{n}=0 \\
-\partial_{z} \tilde{p}^{n}+\left[-\frac{R e}{\Lambda_{a}}\left(s+\Lambda_{n} \mathrm{i} k z\right)+\frac{\eta^{n}}{\eta^{a}}\left(\partial_{z}^{2}-k^{2}\right)\right] \tilde{v}_{z}^{n}=0
\end{gathered}
$$

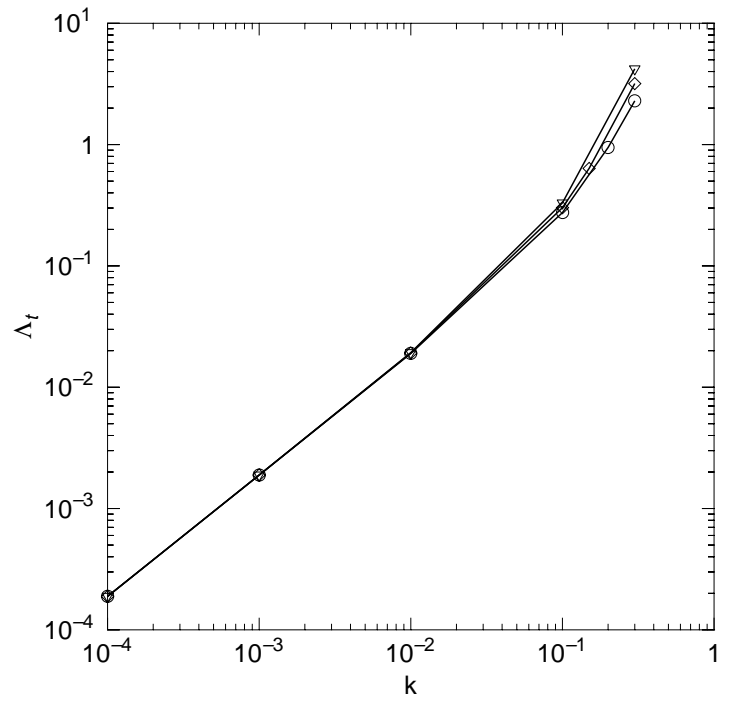

Fig. 6. Variation of transition velocity $\Lambda_{t}$ with $k$ for model B, for different values of (०) $D=0,(\diamond) D=20$, and $(\nabla) D=40$ for fixed $T=100$ and $E=10, B=10^{-2}$.

where $\tilde{p}, \tilde{v}_{x}$ and $\tilde{v}_{z}$ represent the Fourier component of the perturbation to the pressure field, $x$-component velocity and the $z$-component velocity respectively in the fluid.

To obtain the velocity $\tilde{v}_{z}$, eliminate $\tilde{p}$ by combining the $x$ and $z$ momentum equations (47) and (48) and then eliminate $\tilde{v}_{x}$ using continuity (46). The governing equation for $\tilde{v}_{z}$ then reduces to

$$
\left[-\frac{R e}{\Lambda_{a}}\left(s+\Lambda_{n} \mathrm{i} k z\right)+\frac{\eta^{n}}{\eta^{a}}\left(\partial_{z}^{2}-k^{2}\right)\right]\left(\partial_{z}^{2}-k^{2}\right) \tilde{v}_{z}^{n}=0 .
$$

The stress fields are then given by

$$
\tilde{\tau}_{x z}^{n}=\frac{\eta^{n}}{\eta^{a}}\left(\mathrm{i} k \tilde{v}_{z}^{n}+\partial_{z} \tilde{v}_{x}^{n}\right)
$$

and

$$
\tilde{\tau}_{z z}^{n}=-\tilde{p}^{n}+2 \frac{\eta^{n}}{\eta^{a}} \partial_{z} \tilde{v}_{z}^{n}
$$

The boundary conditions at $z=1$ and $z=-H$ for fluids $\mathrm{A}$ and $\mathrm{B}$ respectively are $\tilde{v}_{z}^{n}=0$ and $\tilde{v}_{x}^{n}=0$. The boundary conditions at the interface are (from Eqs. (1517)):

$$
\begin{gathered}
\tilde{v}_{x}^{n}+\Lambda_{n} \frac{\tilde{v}_{z}}{s}=s \tilde{u}_{x} \\
\tilde{v}_{z}^{a}=\tilde{v}_{z}^{b} \\
\tilde{\tau}_{z z}^{a}-\tilde{\tau}_{z z}^{b}=k^{2} \frac{\tilde{v}_{z}}{s} .
\end{gathered}
$$

In the above equation, a term similar to $-2 \Lambda \mathrm{i} k \tilde{u}_{z}$ in (26) has been neglected because the term is $O(k)$ smaller than the right side of the above equation, and this assumption is valid in the low- $k$ limit. The equation for $\tilde{u}_{x}$ is obtained through the force balance (13), and to linear order in the perturbation quantities, is given by:

$$
\tilde{\tau}_{x z}^{a}-\tilde{\tau}_{x z}^{b}-\left(2\left(G+\eta_{\mathrm{m}} s\right)+K\right) k^{2} \tilde{u}_{x}=0 .
$$


The $4 \times 4$ matrix of boundary conditions is assembled, and the determinant set to zero to obtain the growth rate. The neutral stability curve is then obtained by setting the real part of the growth rate to zero. A low Reynolds number analysis is first used to study the stability of the system in the absence of fluid inertia. This results of this analysis shows that the system is unstable even in the absence of inertia. The effect of inertia on the low Reynolds number unstable modes is then examined using a low wave number analysis, which shows that in the presence of inertia the system is still unstable but instability is restricted to a range of Reynolds number. The low Reynolds number modes are later extended numerically to intermediate Reynolds number to compare the instability obtained in the present study with that of the finite Reynolds number instability for incompressible membranes analysed by Kumaran and Srivatsan [12].

\subsection{Low Reynolds number analysis}

The solutions for $\tilde{v}_{z}^{a}$ and $\tilde{v}_{z}^{b}$ for the case $R e=0$ are obtained by inserting $R e=0$ in (49) and satisfying the boundary conditions at $z=1$ and $z=-H$. These eigenfunctions are

$$
\begin{aligned}
\tilde{v}_{z}^{a}= & a_{1}\left[\mathrm{e}^{-k z}-(1+2 k-2 k z) \mathrm{e}^{k z-2 k}\right] \\
& +a_{2}\left[z \mathrm{e}^{-k z}+(2 k z-2 k-z) \mathrm{e}^{k z-2 k}\right] \\
\tilde{v}_{z}^{b}= & b_{1}\left[z \mathrm{e}^{k z}+\left((2 k H-1) \mathrm{e}^{k z}+\mathrm{e}^{-(k z+2 k H)}\right) /(2 k)\right] \\
& +b_{2}\left[z \mathrm{e}^{-k z}+\left((2 k H+1) \mathrm{e}^{-k z}-\mathrm{e}^{k z+2 k H}\right) /(2 k)\right]
\end{aligned}
$$

where $a_{1}, a_{2}, b_{1}$ and $b_{2}$ are constants to be determined from the interface conditions. The tangential velocities $\tilde{v}_{x}^{a}$ and $\tilde{v}_{x}^{b}$ are obtained from the respective continuity equations. Similarly the pressures $\tilde{p}^{a}$ and $\tilde{p}^{b}$ are obtained from the respective $x$-direction momentum balance equations. The boundary conditions at the interface (52-55) can be expressed as a matrix equation of the form

$$
\mathbf{A} \cdot \mathbf{C}=\mathbf{0}
$$

where $\mathbf{C}=\left(a_{1} a_{2} b_{1} b_{2}\right)$ is the vector of the constants, and $\mathbf{A}$ is a $4 \times 4$ matrix. The determinant of matrix $\mathbf{A}$ is then set to zero to obtain the characteristic equation, and the growth rate is obtained by solving this algebraic equation. The expression for the growth rate is not provided here because it is very lengthy, but it is easily obtained using symbolic computation.

The results show that for the case of compressible membranes with finite bulk and shear modulus there exists an instability even at zero Reynolds number. Figures $(7-10)$, show the neutral stability curves in the $\Lambda_{t}-k$ plane for the compressible membrane for various membrane parameters, where $\Lambda_{t}$ is the upper plate velocity required for destabilising the flow. In these figures, the region above the neutral stability curve is unstable while the region below is stable. There is no instability, however, when the viscosity ratio is equal to 1 . The results also

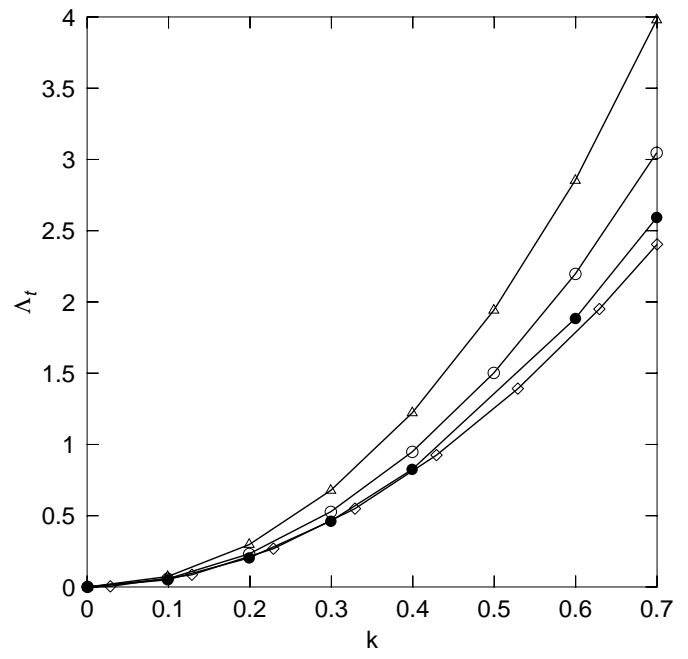

Fig. 7. Variation of transition velocity $\Lambda_{t}$ with wave number $k$ for different relative viscosity of the two fluids in the $R e=0$ limit. $\left((\triangle) \quad \eta^{r}=0.7,(\bigcirc) \quad \eta^{r}=0.5,(\bullet) \eta^{r}=0.3,(\diamond) \eta^{r}=\right.$ $\left.0.1, K=100.0, G=1.0, \eta_{\mathrm{m}}=0, H=1.0\right)$.

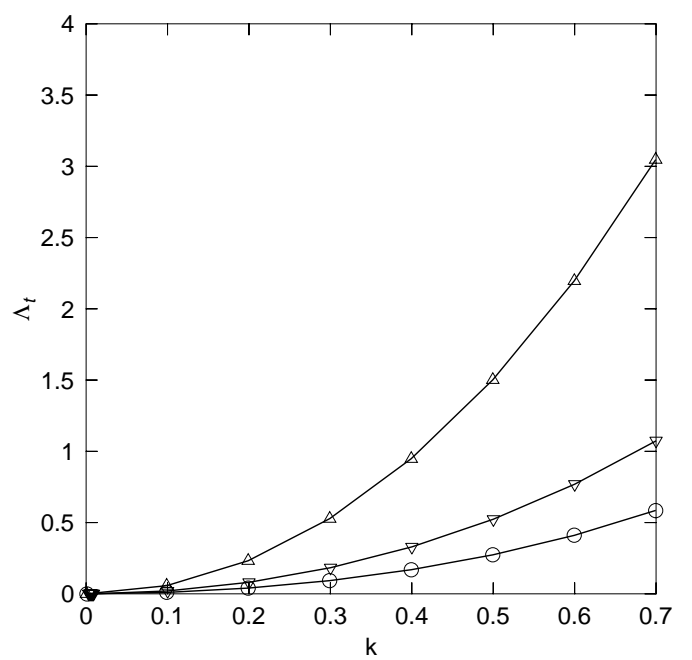

Fig. 8. Variation of transition velocity $\Lambda_{t}$ with wave number $k$ for different bulk modulus of the membrane in the $R e=0$ limit. $(\triangle) \quad K=100,(\nabla) \quad K=10,(\bigcirc) \quad K=1, \eta^{r}=$ $0.5, \eta_{\mathrm{m}}=0, G=1.0, H=1.0$.

show that the nondimensional fluid velocity $\Lambda_{a}$ for unstable fluctuations scales as $O\left(k^{2}\right)$, unlike $O(k)$ behaviour of the neutral modes in the plate-membrane model.

An increase in the relative fluid viscosity (Fig. 7), bulk modulus (Fig. 8) and shear moduli (Fig. 9) results in an increase in the transition velocity, and have a stabilising effect on the system. Thus while the fluid viscosity is stabilising, the increased tangential motion in the membrane due to the decrease in the bulk and shear moduli is destabilising. Figure 11 shows the effect of membrane viscosity on the neutral stability curve. This figure shows that the low- $k$ instability is not affected by the membrane viscosity, and for $k \sim O(1)$, an increase in the membrane viscosity has a stabilising effect on the perturbations. The effect of the ratio of fluid thickness $H$ on the instability is shown in Figure 10. This figure 


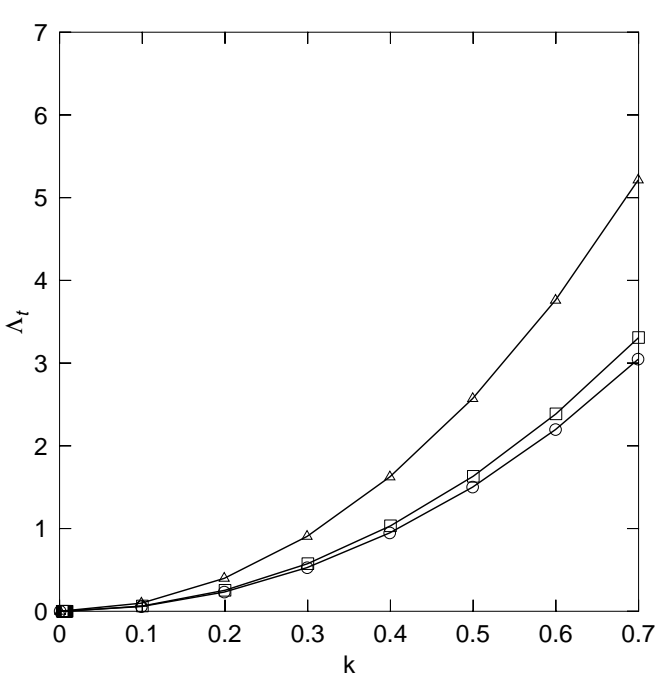

Fig. 9. Variation of transition velocity $\Lambda_{t}$ with wave number $k$ for different shear modulus in the $R e=0$ limit. $(\bigcirc) \quad G=$ $1,(\square) \quad G=10,(\triangle) \quad G=100, \eta^{r}=0.5, \eta_{\mathrm{m}}=0, K=$ $100, H=1.0$.

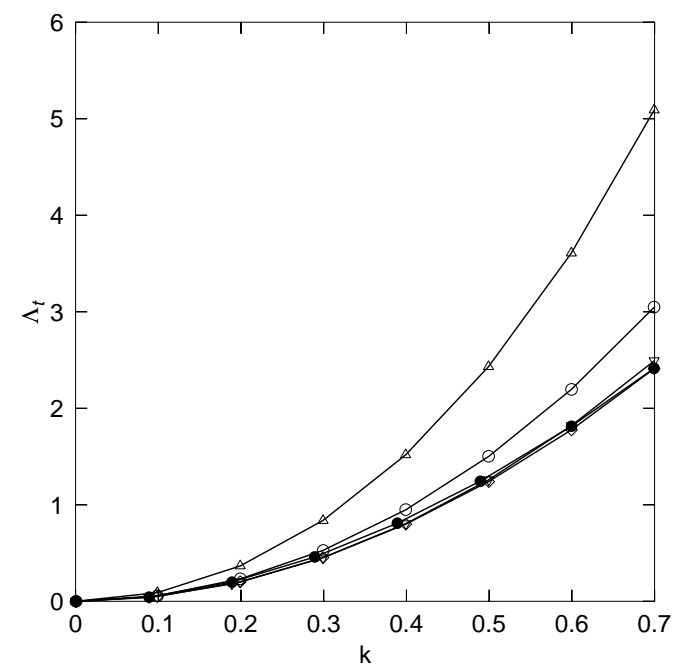

Fig. 10. Variation of transition velocity $\Lambda_{t}$ with wave number $k$ for different $H$ in the $R e=0$ limit. $(\triangle) H=0.8,(\bigcirc)$ $H=1.0,(\nabla) \quad H=1.4,(\diamond) \quad H=1.8,(\bullet) H=5.0, \eta^{r}=$ $0.5, \eta_{\mathrm{m}}=0, K=100.0, G=1.0$.

shows that as the value of $H$ is increased, the system becomes more unstable and at higher $H$ values all the neutral stability curves merge into one, indicating no further effect of $H$ on the neutral modes. The expression for the transition velocity for $\eta_{r}=1 / 2$ can be written as

$$
\Lambda_{t}^{\prime}=\frac{\left(1+2 H^{3}\right) \sqrt{K}}{\left[3 H\left(4 H^{5}+4 a H^{3}+8 H^{2}-3 H-4\right)\right]^{1 / 2}},
$$

where $\Lambda_{t}^{\prime}=\Lambda_{t} / \alpha^{2}$.

The expression for $\Lambda_{t}$ in the limit of $H \gg 1$ is given by $\frac{\sqrt{K}}{2 \sqrt{3}}\left(2-\frac{1}{H^{2}}\right)$ while the critical bottom fluid thickness below which the system is always stable is $\frac{1}{\sqrt{2}}$.

It is instructive to obtain from the present analysis, the asymptotic limit in which the membrane is not present at

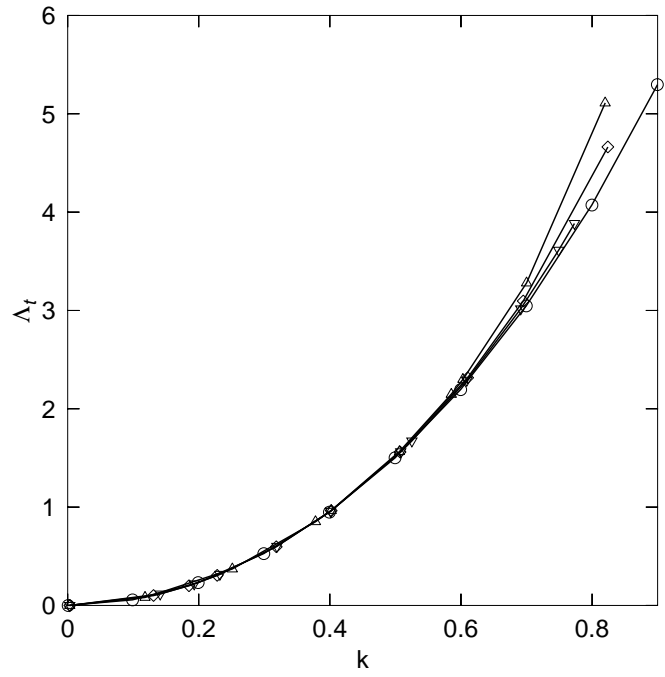

Fig. 11. Variation of transition velocity $\Lambda_{t}$ with wave number $k$ for different membrane viscosity in the $R e=0$ limit. $(\bigcirc)$ $\eta_{\mathrm{m}}=0,(\nabla) \quad \eta_{\mathrm{m}}=10,(\diamond) \quad \eta_{\mathrm{m}}=15,(\triangle) \quad \eta_{\mathrm{m}}=25, G=$ $1, \eta^{r}=0.5, K=100, H=1.0$.

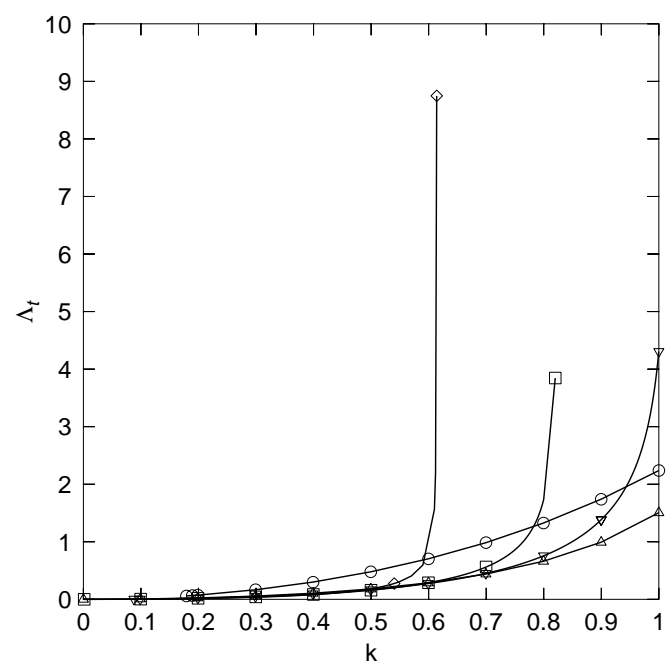

Fig. 12. Variation of transition velocity $\Lambda_{t}$ with wave number $k$ as system approaches no membrane case in the $R e=0$ limit. $(\diamond) K=0.3,(\square) K=0.5,(\nabla) K=0.7,(\triangle) K=1.0$, (○) $K=10, \eta^{r}=0.5, \eta_{\mathrm{m}}=0, H=1.0, G=0.0$.

the interface, where the system consists two Newtonian fluids shearing past each other. It is known that in the low Reynolds number limit the Couette flow of two fluids without a membrane is always stable in the presence of surface tension [17]. There is another asymptotic limit, namely that of an incompressible membrane studied by Kumaran and Srivatsan [12], which can also be recovered from the present analysis as a special limiting case. The stable modes of incompressible membrane are captured by this model as a limiting case of the bulk modulus $K$ and the shear modulus $G \rightarrow \infty$. The no membrane case (just two Newtonian fluids shearing past each other) is obtained by setting the bulk and shear moduli of the membrane equal to zero. The results for this limit are shown in Figure 12, where the transition velocities are shown as 


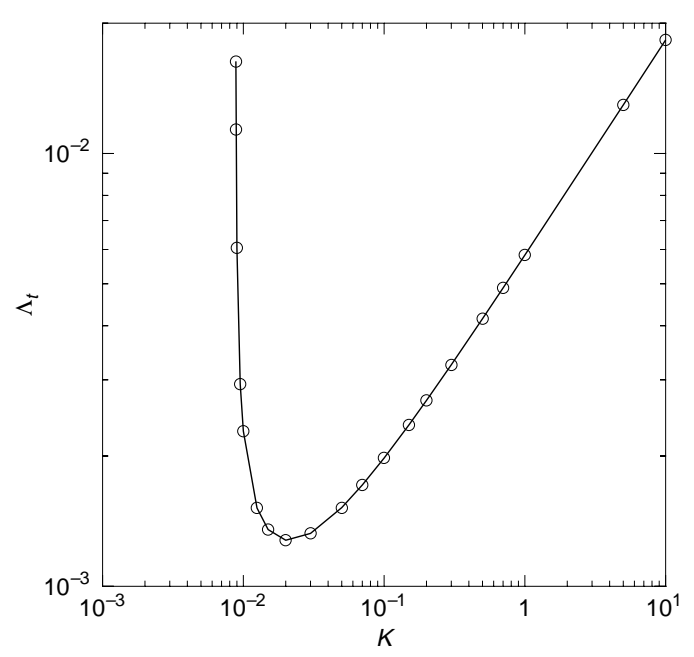

Fig. 13. Variation of transition velocity $\Lambda_{t}$ with bulk modulus $K$ as system approaches no membrane and the incompressible membrane case in the $R e=0$ limit. $\eta^{r}=0.5, \eta_{\mathrm{m}}=0, H=$ $1.0, G=0.0, k=0.1$.

a function of the bulk modulus at zero shear modulus. It is seen that a decrease in the bulk modulus initially destabilises the perturbations, but a further decrease stabilises the system and the instability is restricted to a smaller range of wave numbers. This range of unstable wave numbers goes to zero for $K \rightarrow 0$. Thus, the results of system of Couette flow of two fluids without a membrane in the $R e \rightarrow 0$ limit are recovered in the limit of $G$ and $K \rightarrow 0$. In Figure 13, the variation of the transition velocity $\Lambda_{t}$ is shown as a function of the bulk modulus $K$ of the membrane, for the specific case of $G=0$ and for a given wavenumber $k=0.1$. As this figure shows, the transition velocity required for unstable perturbations diverges both in the $K \ll$ (no membrane case) and $K \gg 1$ (incompressible membrane case), and this behaviour is consistent with the earlier results $[12,17]$. The effect of inertia on the growth rate is examined using a low wavenumber analysis in the next section.

\subsection{Low wavenumber analysis}

In this section, the effect of inertia is incorporated in the limit of $k \rightarrow 0$ using an asymptotic analysis. The low wave number analysis indicates the presence of two transition curves in the $R e-\Sigma$ plane, as shown in Figures 14 and 15 , where $\Sigma=\rho \Gamma R /\left(\eta^{a}\right)^{2}$ is only a function of the fluid and membrane parameters and is independent of the fluid velocity. The lower curve converges to the unstable modes obtained in the limit of zero Reynolds number, and there is a transition from stable to unstable modes as the Reynolds number is increased in this case. However, there is another transition curve above this where there is a transition from unstable to stable modes, indicating that unstable modes are encountered only in a finite domain in the $R e-\Sigma$ plane.

The lower branch is a continuation of the $R e \rightarrow 0$ viscous modes (discussed in the previous section) and hence

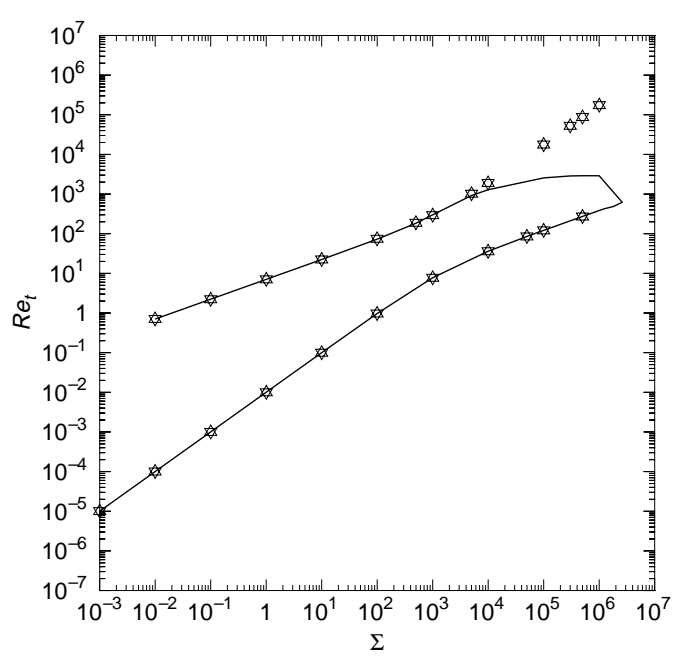

Fig. 14. Comparison of asymptotic and numerical results for variation of transition Reynolds number $R e_{t}$ with $\Sigma$ in the intermediate $R e$ regime. (-) Numerical $R e_{t}, \quad(\star)$ Asymptotic $R e_{t}, G=1.0, K=1.0, \eta_{\mathrm{m}}=0, \eta^{r}=0.5, H=1.0, k=0.1$.

the scalings for the $R e \rightarrow 0$ modes can be used to determine the effect of inertia. Thus, for the lower branch in the limit of $k \ll 1$, if $\tilde{v}_{x}$ is of $O(1)$ then $\tilde{v}_{z}$ is $O(k)$ due to continuity equation and pressure is $O\left(k^{-1}\right)$ from the $x$ momentum equation. Inertial effects in the governing equations of the two fluids are of $O(k R e)$ in the limit of low $k$, and when $R e \sim O(1)$, the inertial stresses can be incorporated through a low wavenumber asymptotic analysis. The interface boundary conditions require $\Lambda_{t}$ to scale as $O\left(k^{2}\right)$ in order for the tangential motion in the membrane to be important. Thus, when expanded in an asymptotic series in $k$, the first two terms in the expression consistent with scaling considerations are:

$$
\begin{aligned}
s & =k^{3}\left(s^{0}+k s^{1}\right)+\ldots, \\
\tilde{v}_{z}^{n} & =k \tilde{v}_{z}^{n 0}+k^{2} \tilde{v}_{z}^{n 1}+\ldots, \\
\tilde{v}_{x}^{n} & =\tilde{v}_{x}^{n 0}+k \tilde{v}_{x}^{n 1}+\ldots, \\
\tilde{p}^{n} & =k^{-1} \tilde{p}^{n 0}+\tilde{p}^{n 1}+\ldots, \\
\Lambda_{n} & =k^{2} \Lambda_{n}^{0}+\ldots
\end{aligned}
$$

where $\Lambda_{n}^{0}$ is $O(1)$.

The above expressions are inserted in the governing equations and solved at each order of $k$ to obtain the leading order and first order corrections to the velocities and pressure as well as the growth rate. The expressions for growth rate arising out of this analysis are lengthy and are presented in Appendix C. The analysis reveals that the leading order growth rate $s^{0}$ is imaginary, while the first correction to growth rate $s^{1}$ is real. The expression for $s^{1}$ shows that inertia has a destabilising effect for the lower branch. The neutral curve for these modes can be obtained by setting $s^{1}$ to zero. The expression for neutral curve for the lower branch shows that $R e \sim \Sigma$ at low values of $\Sigma$, while it goes as $\Sigma^{0.5}$ at high values of $\Sigma$. The results of asymptotic analysis compare well with the numerical results as shown in Figures 14 and 15. 


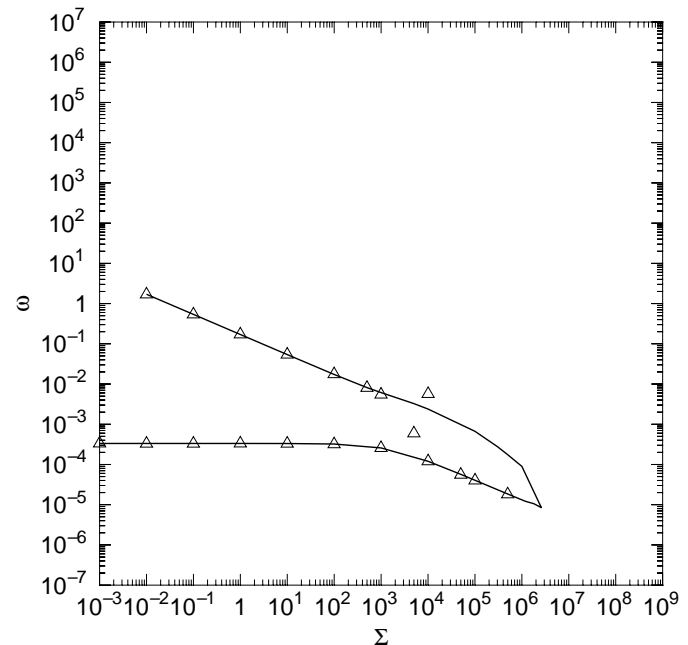

Fig. 15. Comparison of asymptotic and numerical results for the frequency $\omega$ at transition with $\Sigma$ in the intermediate $R e$ regime. (-) Numerical, $(\triangle)$ Asymptotic $\omega, G=1.0, K=$ $1.0, \eta_{\mathrm{m}}=0, \eta^{r}=0.5, H=1.0, k=0.1$.

The upper branch is also accessible to low wavenumber asymptotic analysis as it exists at Reynolds numbers of $O(1)$. The scalings for the pressure and velocity are same as for the lower branch, but the scalings for the growth rate and the strain rate are:

$$
\begin{aligned}
s & =k\left(s^{0}+k s^{1}\right)+\ldots, \\
\Lambda_{a} & =\Lambda_{a}^{0}+\ldots
\end{aligned}
$$

The above expressions, when inserted in the governing equations, yield the values of the leading order growth rate $s^{0}$ and the first order correction $s^{1}$. The analysis shows that $s^{0}$ is purely imaginary, but $s^{1}$ is real, and the expression for $s^{1}$ shows that inertia has a stabilising effect. The neutral curve can again be obtained by setting $s^{1}=0$. The expression for the neutral curve, provided in Appendix C, shows that $R e \sim \Sigma^{0.5}$ for the neutral modes and this compares well with the numerical result (see Figs. 14 and 15). It is important to note here that the above analysis and results are valid only when $\Lambda_{t}$ is $O(1)$, because it is assumed in the analysis that the tension due to mean flow is small compared to the intrinsic and imposed surface tension.

\subsection{Intermediate Reynolds number regime}

The governing equation for $\tilde{v}_{z}$ at finite $R e$ is a linear fourth order ordinary differential equation with variable coefficients. This is reduced to an Airy type of equation which however is solved numerically, using a method similar to that of Srivatsan and Kumaran [9]. There are four constants of integration for the fourth order differential equation, out of which two are fixed by the zero velocity condition at $z=1$ and $z=-H$. Consequently, there are two linearly independent solutions for the velocity fields of fluids A and B. The fourth order differential equations for the velocity fields are solved using a fourth order RungeKutta integrator. The values for the fundamental solutions

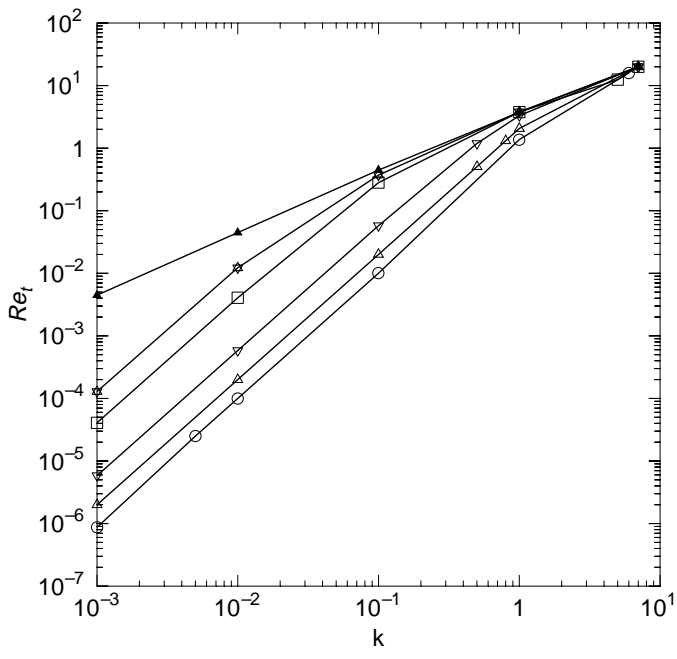

Fig. 16. Variation of transition Reynolds number $R e_{t}$ with wave number $k$ for different bulk moduli in the intermediate $R e$ regime. ( $\mathbf{\Delta})$ Incompressible, $(\bigcirc) K=1,(\triangle) K=10.0,(\nabla)$ $K=100.0,(\square) \quad K=5000,(\star) K=50000, G=1.0, \eta^{r}=$ $0.5, \eta_{\mathrm{m}}=0, H=1.0, \Sigma=1.0$.

at the membrane surface were inserted into the boundary conditions and a $4 \times 4$ characteristic matrix is formed. The characteristic equation is obtained by setting the determinant of the this matrix equal to zero. The characteristic equation is nonlinear in the growth rate $s$, and a Newton Raphson iteration scheme was used to get the roots of characteristic equation, with the analytical results for $R e=0$ used as the starting guess.

The numerical method for solving the governing stability equations was validated with the analytical solutions for low $R e$ limit as well as the low wavenumber asymptotic results. The present numerical method was also validated with the results of Kumaran and Srivatsan [12] for the case of the incompressible membrane, in the limit of $K \rightarrow \infty$. Figures 14 and 15 show the comparison between the numerical results and low wavenumber analysis. As can be seen, the numerical results match well with the low wavenumber asymptotic results when $R e$ is $O(1)$. At higher $R e$ the deviation increases and it is necessary to include higher order corrections.

Results for neutral stability are plotted in Figure 16 for transition Reynolds number as a function of the wavenumber and the dimensionless parameter $\Sigma=R e / \Lambda_{a}=\rho \Gamma R /\left(\eta^{a}\right)^{2}$. The figures shows that the transition Reynolds number increases proportional to $k^{2}$ when the bulk modulus is finite, but it approaches the $R e_{t} \propto k$ behaviour of Kumaran and Srivatsan [12] for $K \rightarrow \infty$. Figure 17 shows that the frequency $\omega$ is positive, indicating that the unstable waves travel downstream, and the frequency increases with an increase in $R e_{t}$. The unstable waves are downstream travelling in all the cases studied.

Figure 14 shows neutral stability curve in the $R e-\Sigma$ plane which has two branches and encloses a finite region of unstable modes. This 'upper branch' of the neutral stability curve in the $R e-\Sigma$ plane is accessible to the low $k$ asymptotic analysis results when the Reynolds 


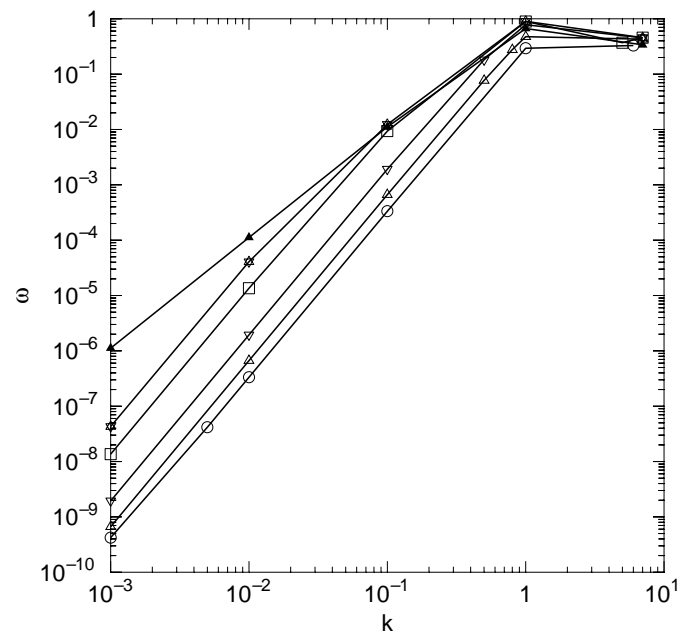

Fig. 17. Variation of frequency $\omega$ for downstream travelling waves with wave number $k$ for different bulk moduli in the intermediate Re regime. (ム) Incompressible, $(\bigcirc) \quad K=1$, $(\triangle) K=10.0,(\nabla) \quad K=100.0,(\square) K=5000,(\star) K=$ $50000, G=1.0, \eta^{r}=0.5, \eta_{\mathrm{m}}=0.0, H=1.0, \Sigma=1.0$.

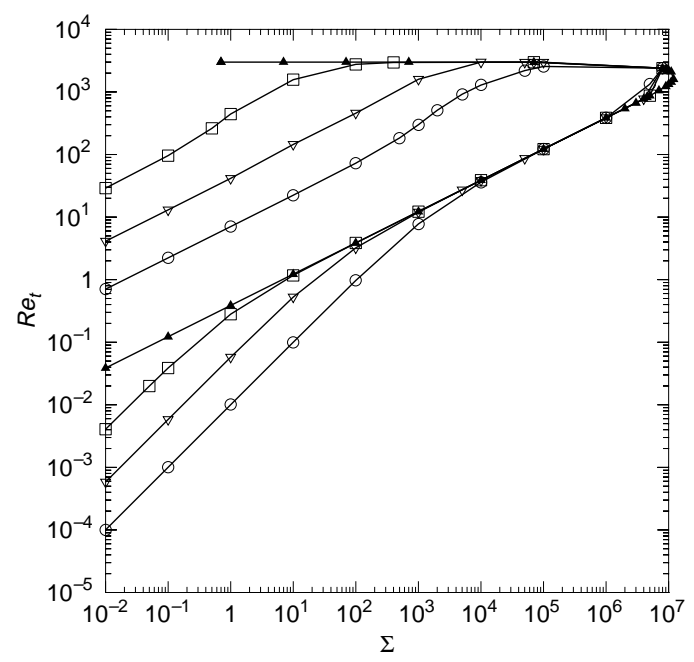

Fig. 18. Variation of transition Reynolds number $R e_{t}$ with $\Sigma$ for different bulk moduli in the intermediate Re regime. ( $\mathbf{\Lambda})$ Incompressible, $(\bigcirc) K=1,(\nabla) K=100.0,(\square) \quad K=5000$, $G=1.0, \eta^{r}=0.5, \eta_{\mathrm{m}}=0, H=1.0, k=0.1$.

number is $O(1)$. Thus for a membrane with given $\Sigma$, as the Reynolds number is increased, the system goes from stable to unstable region as we cross the "lower branch". There is again a transition from unstable to stable modes when $R e$ is increased beyond the upper branch.

The effect of bulk modulus is shown in Figures 18 and 19. These figures indicate that the neutral stability curves approach the result of Kumaran and Srivatsan [12] with increasing bulk modulus, as should be expected. The $R e-\Sigma$ plot shows that the transition Reynolds number increases with $\Sigma$. While $R e_{t}$ is $\propto$ to $\Sigma$ at low $\Sigma$ values, it scales as $\Sigma^{0.5}$ at higher values of $\Sigma$. The curve then turns back after certain $\Sigma$ ("upper branch") beyond which the flow is always stable. Thus while inertial effects are destabilising in the lower branch, they have a stabilising effect in the upper branch. The $\Lambda_{t}-\Sigma$ curves (Fig. 20) show

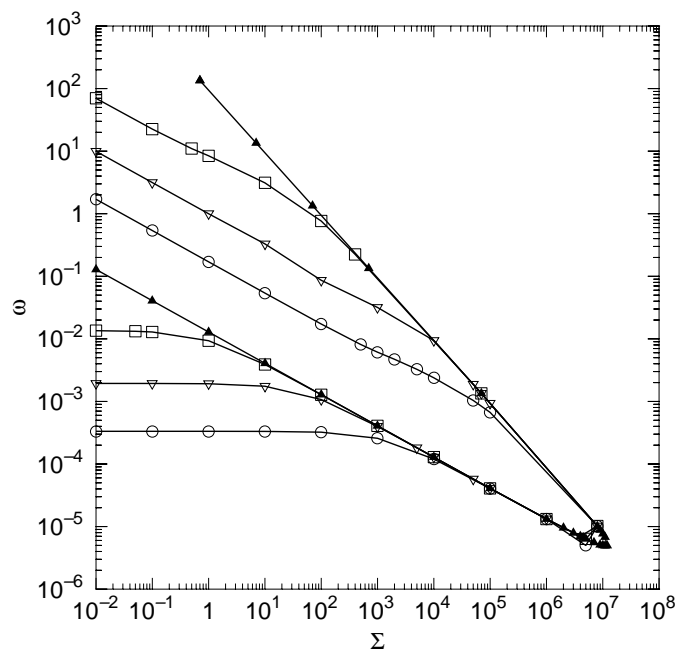

Fig. 19. Variation of frequency $\omega$ with $\Sigma$ for different bulk moduli in the intermediate Re regime. ( $\mathbf{\Lambda})$ Incompressible, $(\bigcirc)$ $K=1,(\nabla) K=100.0,(\square) \quad K=5000, G=1.0, \eta^{r}=0.5$, $\eta_{\mathrm{m}}=0, H=1.0, k=0.1$.

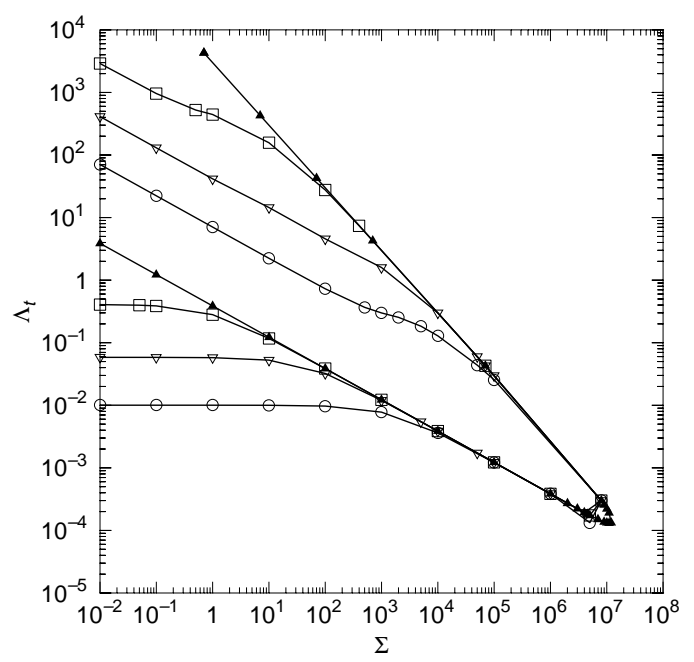

Fig. 20. Variation of transition velocity $\Lambda_{t}$ with $\Sigma$ for different bulk moduli in the intermediate $R e$ regime. ( $\mathbf{\Lambda})$ Incompressible, $(\bigcirc) \quad K=1,(\nabla) \quad K=100.0,(\square) K=5000, G=$ $1.0, \eta^{r}=0.5, \eta_{\mathrm{m}}=0, H=1.0, k=0.1$.

that the transition velocity $\Lambda_{t}$ is constant for the lower branch and is small compared to unity. However for low values of $\Sigma$, in the upper branch $\Lambda_{t} \gg 1$ and hence the results in this region should have corrections due to mean flow tension, as discussed in Section 2.2.

\section{Conclusions}

The stability of linear shear flow of a Newtonian fluid of thickness $R$ past a flexible membrane was analysed in the limit of low Reynolds number as well as in the intermediate Reynolds number regime for two different membrane models. This work demonstrates the importance of tangential motion in the membrane on the stability characteristics of the shear flow in the low Reynolds number limit. The first model assumes the wall to be a simple plate 
membrane and the displacement of the wall is phenomenologically related in a linear manner to the fluid stresses at the wall. In the second model, the membrane is assumed to be a two-dimensional compressible viscoelastic solid continuum of infinitesimal thickness, but where the normal stress is proportional to the normal displacement instead of the curvature, as applicable to anchored membranes. It is seen that the stability characteristics of the laminar flow are sensitive to the wall model used. In particular, it is found that for both the cases, the shear flow is stable in the low Reynolds number limit in the absence of tangential motion in the membrane. However, the presence of tangential motion in the membrane destabilises the shear flow even in the absence of fluid inertia. It is also observed that the low wave number characteristics of model B and a viscoelastic membrane are similar. This indicates that the instability is driven primarily by the tangential deformation, since the constitutive relations for the tangential deformation for the two cases are identical.

In the case of the viscoelastic membrane model, it is found that the flow past a compressible membrane could be unstable in the zero Reynolds number limit, while the limiting cases of an incompressible membrane (large bulk modulus) and the interface between two fluids (zero shear and bulk moduli) without a membrane are both stable in this limit. Thus, the compressibility of the membrane appears to qualitatively alter the stability of the flow in the limit of low Re. The results of the low Reynolds number analysis were extended numerically to the intermediate Reynolds number regime for the case of the viscoelastic membrane. The results from the numerical calculations for the viscoelastic membrane reveal that the instability in this regime for compressible membrane asymptotically approach the previous results of Kumaran and Srivatsan [12] for the incompressible membrane in the limit of large bulk modulus. The Reynolds number for neutral modes scales as $\Sigma^{1 / 2}$ for the incompressible membrane as reported by Kumaran and Srivatsan [12]. The present results, however, show that $R e \sim \Sigma$ for low $\Sigma$, and it crosses over to $R e \sim \Sigma^{1 / 2}$ for higher values of $\Sigma$, thus demonstrating that the instability analysed here is different from that of Kumaran and Srivatsan [12].

It is useful to examine whether the instability predicted here could be observed for parameters of interest in real biological systems. Using estimates, $K^{*}=10^{-1} \mathrm{~N} / \mathrm{m}$ and a tension of the order of $\Gamma=10^{-3} \mathrm{~N} / \mathrm{m}$ corresponding to a linear extension ratio of $1 \%$, the nondimensional elasticity is of the order of $K=K^{*} / \Gamma^{*} \sim$ 100 . The non-dimensional strain rate causing instability $\Lambda \sim \alpha^{2}(\Gamma / \eta R)$. For realistic values of $\eta=10^{-4} \mathrm{~kg} / \mathrm{m} / \mathrm{s}$, $\Gamma=10^{-3} \mathrm{~N} / \mathrm{m}$ and $R=10^{-4} \mathrm{~m}$, and $\alpha \sim 10^{-2}$ corresponding to wavelengths of around 100 times the channel width, $\Lambda \sim 10 \mathrm{~s}^{-1}$. This corresponds to a maximum velocity of $10^{-3} \mathrm{~m} / \mathrm{s}$ in a channel of width $10^{-4} \mathrm{~m}$, and this is certainly in the range of velocities encountered in biological systems.

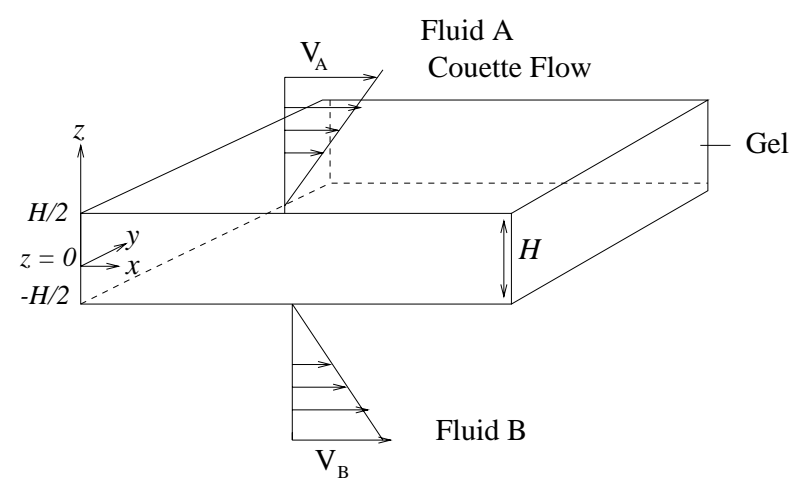

Fig. 21. Configuration to illustrate the derivation of infinitesimal thin membrane equation.

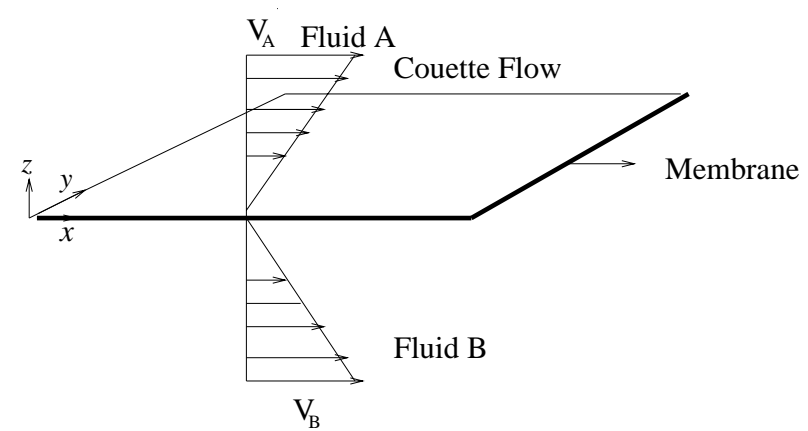

Fig. 22. Configuration for the two-dimensional membrane.

\section{Appendix A: Derivation of force balance equation}

Here we present a brief derivation on how the membrane constitutive relation and the interface force balance conditions (13) arise, as a $H \rightarrow 0$ limiting case of a viscoelastic compressible gel of finite thickness $H$. As shown below, the stress condition used in Section 2.2 arises from balancing the fluid tractions at the two sides of the gel with the gel tractions.

Consider a viscoelastic gel of finite thickness $H$ with the origin placed at the centre of the gel, as illustrated in Figure 21. As the figure shows, $x$ and $y$ are the inplane coordinates in the rectangular slab, and $z$ is the normal Cartesian coordinate. Let $\sigma_{i j}^{*}$ be the gel stress tensor in this three dimensional continuum, with dimensions Force/Area. The governing equation for the gel, in the limit of zero inertia in the gel, is given by

$$
\partial_{j} \sigma_{i j}^{*}=0
$$

The $x$-momentum balance takes the following form

$$
\frac{\partial \sigma_{x x}^{*}}{\partial x}+\frac{\partial \sigma_{x y}^{*}}{\partial y}+\frac{\partial \sigma_{x z}^{*}}{\partial z}=0 .
$$

When the thickness of the wall medium is small compared to the thickness of the fluid layer and the amplitude of fluctuations, one is not interested in the variations of the dynamical quantities along $z$-direction in the gel wall, and it is appropriate to integrate the variations in the $z$-direction of the gel medium. Accordingly, the above stress balances 
can be integrated in the $z$-direction from $z=-H / 2$ to $z=H / 2$ to give:

$$
\int_{-H / 2}^{H / 2}\left(\frac{\partial \sigma_{x x}^{*}}{\partial x}+\frac{\partial \sigma_{x y}^{*}}{\partial y}+\frac{\partial \sigma_{x z}^{*}}{\partial z}\right) \mathrm{d} z=0 .
$$

The height averaged quantities are defined as:

$$
\langle f(x, y)\rangle=\frac{1}{H} \int_{-H / 2}^{H / 2} f(x, y, z) \mathrm{d} z .
$$

It should be noted here that once the dynamical quantities are integrated over the thickness of the gel (i.e. in the $z$ direction), clearly they are no longer dependent on the $z$ variable, as shown in Figure 22. Then (63) becomes

$$
\begin{aligned}
H \frac{\partial\left\langle\sigma_{x x}^{*}\right\rangle}{\partial x}+ & H \frac{\partial\left\langle\sigma_{x y}^{*}\right\rangle}{\partial y} \\
& +\left(\left.\left\langle\sigma_{x z}^{*}\right\rangle\right|_{z=H / 2}-\left.\left\langle\sigma_{x z}^{*}\right\rangle\right|_{z=-H / 2}\right)=0 .
\end{aligned}
$$

The traction continuity conditions at $z=H / 2$ and $z=$ $-H / 2$ yields the following:

$$
\begin{aligned}
\left.\left\langle\sigma_{x z}^{*}\right\rangle\right|_{H / 2} & =\tau_{x z}^{\mathrm{A}} \\
\left.\left\langle\sigma_{x z}^{*}\right\rangle\right|_{-H / 2} & =\tau_{x z}^{\mathrm{B}} .
\end{aligned}
$$

Here $\tau_{x z}^{\mathrm{A}}$ and $\tau_{x z}^{\mathrm{B}}$ are fluid shear stresses exerted respectively by the top (A) and bottom (B) fluids on the gel. Redefining the height averaged gel stresses as

$$
H\left\langle\sigma_{x x}^{*}\right\rangle=\sigma_{x x}^{\mathrm{m}}, \quad H\left\langle\sigma_{x y}^{*}\right\rangle=\sigma_{x y}^{\mathrm{m}}
$$

where $\sigma_{x x}^{\mathrm{m}}$ and $\sigma_{x y}^{\mathrm{m}}$ are now the membrane stresses, we obtain from (65):

$$
\frac{\partial \sigma_{x x}^{\mathrm{m}}}{\partial x}+\frac{\partial \sigma_{x y}^{\mathrm{m}}}{\partial y}+\tau_{x z}^{\mathrm{A}}-\tau_{x z}^{\mathrm{B}}=0
$$

and

$$
\frac{\partial \sigma_{x y}^{\mathrm{m}}}{\partial x}+\frac{\partial \sigma_{y y}^{\mathrm{m}}}{\partial y}+\tau_{y z}^{\mathrm{A}}-\tau_{y z}^{\mathrm{B}}=0 .
$$

Thus while the gel stresses $\sigma_{i j}^{*}$ have the dimensions of Force/area, the membrane stresses $\sigma_{i j}^{\mathrm{m}}$ which are averaged over the thickness of the membrane (see (68)) have the dimensions of Force/length.

\section{Appendix B: Squire's theorem}

It is shown here that the Squire's theorem, which states that two-dimensional perturbations are always more unstable than the three-dimensional ones, is valid for the flow past a compressible membrane with surface tension in the limit of zero Reynolds number.

The generalisation of equation (13) for three dimensional disturbances, after nondimensionalising using the scales given in Section 2.2, is given by:

$$
\begin{aligned}
\tau_{x z}^{a}-\tau_{x z}^{b} & +\left(G+\eta_{\mathrm{m}} \partial_{t}\right)\left(\partial_{x}^{2}+\partial_{y}^{2}\right) u_{x} \\
& \left.+\left(G+\eta_{\mathrm{m}} \partial_{t}+K\right)\left(\partial_{x}\left(\partial_{x} u_{x}+\partial_{y} u_{y}\right)\right)\right]=0
\end{aligned}
$$

$$
\begin{aligned}
\tau_{y z}^{a}-\tau_{y z}^{b} & +\left[\left(G+\eta_{\mathrm{m}} \partial_{t}\right)\left(\partial_{x}^{2}+\partial_{y}^{2}\right) u_{y}\right. \\
& \left.+\left(G+\eta_{\mathrm{m}} \partial_{t}+K\right)\left(\partial_{y}\left(\partial_{x} u_{x}+\partial_{y} u_{y}\right)\right)\right]=0
\end{aligned}
$$

where the parameters are as defined earlier. The normal stress condition at the interface is

$$
\tau_{z z}^{a}-\tau_{z z}^{b}=-\left(\partial_{x}^{2}+\partial_{y}^{2}\right) u_{x}
$$

The equations of motion for the fluid in the zero Reynolds number limit, scaled as in Section 2.2, are

$$
\begin{gathered}
\partial_{i} v_{i}^{n}=0 \\
-\partial_{i} p^{n}+\frac{\eta^{n}}{\eta^{a}} \partial_{j}^{2} v_{i}^{n}=0
\end{gathered}
$$

where $n$ is $\mathrm{A}$ or $\mathrm{B}$ for fluid $\mathrm{A}$ and $\mathrm{B}$ respectively. The boundary conditions at the interface are the continuity of velocities and stresses in the normal and tangential directions. At the upper rigid plate $(z=1)$ and the lower rigid plate $(z=-H)$, no-slip boundary conditions are appropriate for all the components of the fluid velocity. Force balance equations written earlier complete the description of boundary conditions at the interface.

Three-dimensional perturbations of the form

$$
v_{i}=\tilde{v}_{i}(z) \mathrm{e}^{\mathrm{i}(k x+l y)+s t}, \quad u_{i}=\tilde{u}_{i}(z) \mathrm{e}^{\mathrm{i}(k x+l y)+s t}
$$

are imposed on the velocity and displacement fields, where $k$ and $l$ are the wavenumbers in the $x$-and $y$ directions. Substituting the above form for the perturbations into the governing equations for the fluid and the membrane, we obtain the following linearised equations governing the stability in the fluid :

$$
\begin{gathered}
\partial_{z} \tilde{v}_{z}^{n}+\mathrm{i}\left(k \tilde{v}_{x}^{n}+l \tilde{v}_{y}^{n}\right)=0 \\
-\mathrm{i} k \tilde{p}^{n}+\frac{\eta^{n}}{\eta^{a}}\left(\partial_{z}^{2}-\left(k^{2}+l^{2}\right)\right) \tilde{v}_{x}^{n}=0 \\
-\mathrm{i} l \tilde{p}^{n}+\frac{\eta^{n}}{\eta^{a}}\left(\partial_{z}^{2}-\left(k^{2}+l^{2}\right)\right) \tilde{v}_{y}^{n}=0 \\
-\partial_{z} \tilde{p}^{n}+\frac{\eta^{n}}{\eta^{a}}\left(\partial_{z}^{2}-\left(k^{2}+l^{2}\right)\right) \tilde{v}_{z}^{n}=0
\end{gathered}
$$

where $\tilde{p}$ represents the Fourier component of the perturbation to the pressure field in the fluid.

The following transformations are introduced for reducing the above three-dimensional problem to an equivalent two dimensional problem

$$
\begin{aligned}
k^{*} & =\left(k^{2}+l^{2}\right)^{1 / 2} \\
k^{*} \tilde{v}_{x}^{*} & =k \tilde{v}_{x}^{n}+l \tilde{v}_{y}^{n} \\
k^{*} \tilde{u}_{x}^{*} & =k \tilde{u}_{x}+l \tilde{u}_{y} \\
\tilde{p}^{*} & =\tilde{p} \\
\tilde{v}_{z}^{*} & =\tilde{v}_{z} \\
s^{*} & =s \\
k^{*} \Lambda^{*} & =k \Lambda .
\end{aligned}
$$

With the above transformations, the continuity equation (77) reduces to

$$
\partial_{z} \tilde{v}_{z}^{*}+\mathrm{i} k^{*} \tilde{v}_{x}^{*}=0
$$


and the momentum equations (after adding $\mathrm{i} k \times x$ momentum Eq. (78) and il $\times y$-momentum Eq. (79)) reduce to

$$
-\mathrm{i} k^{*} \tilde{p}^{*}+\frac{\eta^{a}}{\eta^{n}}\left(\partial_{z}^{2}-\left(k^{*}\right)^{2}\right) \tilde{v}_{x}^{*}=0
$$

and the $z$-momentum equation (80) reduces to

$$
-\partial_{z} \tilde{p}^{*}+\frac{\eta^{a}}{\eta^{n}}\left(\partial_{z}^{2}-\left(k^{*}\right)^{2}\right) \tilde{v}_{z}^{*}=0
$$

These equations are identical to those for two-dimensional perturbations with wavenumber $k^{*}$ and growth rate $s^{*}$. The normal stress boundary condition is given by:

$$
\tilde{\tau}_{z z}^{a}-\tilde{\tau}_{z z}^{b}=\left(k^{2}+l^{2}\right) \frac{\tilde{v}_{z}^{n}}{s}
$$

where

$$
\tilde{\tau}_{z z}^{n}=\left(-p^{n}+\frac{\eta^{n}}{\eta^{a}} 2 \partial_{z} \tilde{v}_{z}^{n}\right)
$$

transforms to

$$
\left(\tilde{\tau}_{z z}^{*}\right)^{n}=\left(k^{*}\right)^{2} \frac{\left(\tilde{v}_{z}^{*}\right)^{n}}{s^{*}}
$$

where

$$
\left(\tilde{\tau}_{z z}^{*}\right)^{n}=\left(-p^{*}\right)^{n}+\frac{\eta^{n}}{\eta^{a}} 2 \partial_{z}\left(\tilde{v}_{z}^{*}\right)^{n}
$$

The tangential velocity boundary conditions, which are given by

$$
\begin{aligned}
\tilde{v}_{x}^{n}+\Lambda^{n} \frac{\tilde{v}_{z}^{n}}{s} & =s \tilde{u}_{x}^{n} \\
\tilde{v}_{y}^{n} & =s \tilde{u}_{y}^{n}
\end{aligned}
$$

are added to provide the equivalent boundary condition for the two-dimensional problem

$$
\left(\tilde{v}_{x}^{*}\right)^{n}+\left(\Lambda^{*}\right)^{n} \frac{\left(\tilde{v}_{z}^{*}\right)^{n}}{s^{*}}=s^{*}\left(\tilde{u}_{x}^{*}\right)^{n} .
$$

The expressions for the tangential stresses in the fluid,

$$
\begin{gathered}
\tilde{\tau}_{x z}^{n}=\frac{\eta^{n}}{\eta^{a}}\left(\partial_{z} \tilde{v}_{x}^{n}+\mathrm{i} k \tilde{v}_{z}^{n}\right) \\
\tilde{\tau}_{y z}^{n}=\frac{\eta^{n}}{\eta^{a}}\left(\partial_{z} \tilde{v}_{y}^{n}+\mathrm{i} l \tilde{v}_{z}^{n}\right)
\end{gathered}
$$

are reduced to the equivalent tangential stress for the twodimensional problem by adding $k \times \tilde{\tau}_{x z}^{n}+l \times \tilde{\tau}_{y z}^{n}$

$$
\tilde{\tau}_{x z}^{*^{n}}=\left(\partial_{z} \tilde{v}_{x}^{*^{n}}+\mathrm{i} k^{*^{n}} \tilde{v}_{z}^{*^{n}}\right) \frac{\eta^{n}}{\eta^{a}} .
$$

The boundary conditions for the tangential stress,

$$
\begin{aligned}
\tilde{\tau}_{x z}^{a}-\tilde{\tau}_{x z}^{b}-(G & \left.+\eta_{\mathrm{m}} s\right)\left(k^{2}+l^{2}\right) \tilde{u}_{x} \\
& -\left(G+\eta_{\mathrm{m}} s+K\right)\left(k\left(k \tilde{u}_{x}+l \tilde{u}_{y}\right)\right)=0
\end{aligned}
$$

$$
\begin{aligned}
\tilde{\tau}_{y z}^{a}-\tilde{\tau}_{y z}^{b}-\left(G+\eta_{\mathrm{m}} s\right)\left(k^{2}+l^{2}\right) \tilde{u}_{y} \\
-\left(G+\eta_{\mathrm{m}} s+K\right)\left(l\left(k \tilde{u}_{x}+l \tilde{u}_{y}\right)\right)=0
\end{aligned}
$$

are reduced to the equivalent conditions for the two dimensional problem by adding $k \times(99)+l \times(100)$ to provide

$$
\begin{aligned}
\left(\tilde{\tau}_{x z}^{*}\right)^{a}-\left(\tilde{\tau}_{x z}^{*}\right)^{b}- & \left(G+\eta_{\mathrm{m}} s\right)\left(\left(k^{*}\right)^{2}\right) \tilde{u}_{x}^{*} \\
& -\left(G+\eta_{\mathrm{m}} s+K\right)\left(k^{*}\left(k^{*} \tilde{u}_{x}^{*}\right)\right)=0 .
\end{aligned}
$$

The above transformations indicate that if a threedimensional problem, with scaled fluid velocity $\Lambda$ and wave numbers $k$ and $l$ in the flow and vorticity directions, has a growth rate $s$, then there is an equivalent two-dimensional problem with scaled fluid velocity $\Lambda^{*}=$ $\Lambda \frac{k}{\left(k^{2}+l^{2}\right)^{1 / 2}}$ and wave number $k^{*}=\left(k^{2}+l^{2}\right)^{1 / 2}$ which has the same growth rate. For the transition from stable to unstable perturbations, the real part of the growth rate is zero, and this transition takes place at a lower scaled mean velocity $\Lambda^{*}$ for two-dimensional disturbances than the scaled velocity $\Lambda$ for three-dimensional disturbances. This shows that two dimensional disturbances are always more unstable than three dimensional ones for a compressible membrane in the zero Reynolds number limit.

\section{Appendix C: Expressions for growth rate in the low wavenumber limit}

In this appendix, we provide analytical expressions for the growth rates for the low- $k$ expansion carried out in Section 3.4 .

The lower branch: The leading order growth rate for the lower branch, which is the continuation of the $R e \rightarrow 0$ branch is obtained as

$$
s^{0}=\frac{-\mathrm{i}}{2} \frac{\Lambda_{0} H^{3}}{\left(H^{3}+\eta_{r}\right)} .
$$

The expression for the first correction to the growth rate is very lengthy, and hence we present here the expression for the case of $H=1$ and $\eta_{r}=1 / 2$.

$$
s^{1}=\frac{45 \Lambda_{0}^{2}+(B+2 E)\left(-15+\Lambda_{0} R e\right)}{270(B+2 E)} .
$$

The expression for $s^{1}$ shows the destabilising effect of fluid inertia on the lower branch modes. The neutral curves can then be obtained by substituting the real part of $s^{1}$ equal to zero, to get:

$$
R e=\left[\frac{15(B+2 E) \Sigma}{B+2 E+\frac{45}{k^{2} \Sigma}}\right]^{1 / 2} k
$$


which shows that $R e \sim \Sigma$ for low values of $\Sigma$ while it goes as $\Sigma^{0.5}$ for high values of $\Sigma$.

The Upper branch: The leading order growth rate is obtained by substituting the scalings for the upper branch in the governing equations and boundary conditions, which yields

$$
s^{0}=\frac{-2 \mathrm{i} \Lambda_{0} H^{2}(1+H) \eta_{r}}{H^{4}+4 H \eta_{r}+6 H^{2} \eta_{r}+4 H^{3} \eta_{r}+\eta_{r}{ }^{2}} .
$$

The expression for the first correction to the growth rate for the case of $H=1$ and $\eta_{r}=\frac{1}{2}$.

$$
s^{1}=\frac{49005(B+2 E)-2962 \Lambda_{0} R e}{718740} .
$$

This shows that fluid inertia has a stabilising effect on the upper branch modes. The neutral curves are then obtained by substituting the value of $s^{1}$ equal to zero, to get,

$$
R e=\left(\frac{49005}{2962}\right)^{1 / 2}[(B+2 E) \Sigma]^{1 / 2}
$$

which shows that $R e \sim \Sigma^{0.5}$.

\section{References}

1. C. Davies, P.W. Carpenter, J. Fluid. Mech. 352, 205 (1997).

2. P.G. La Rose, J.B. Grotberg, J. Fluid. Mech. 331, 37 (1997).

3. P.W. Carpenter, J.S.B. Gajjar, Theoret. Comput. Fluid Dyn. 1, 349 (1990).

4. P.W. Carpenter, A.D. Garrad, J. Fluid. Mech 170, 199 (1986).

5. J.S.B. Gajjar, S.K. Sibanda, Theor. Comput. Fluid Dyn. 8, 105 (1996).

6. V. Kumaran, G.H. Fredrickson, P. Pincus, J. Phys. II France 4, 893 (1994).

7. V. Kumaran, J. Chem. Phys. 98, 3429 (1993).

8. V. Kumaran, J. Fluid. Mech. 294, 259 (1995).

9. L. Srivatsan, V. Kumaran, J. Phys. II France 7, 947 (1997).

10. V. Kumaran, J. Fluid. Mech 357, 123 (1998).

11. J.L. Harden, H. Pleiner, Phys. Rev. E 49, 1411 (1994).

12. V. Kumaran, L. Srivatsan, Eur. Phys. J. B 2, 259 (1998).

13. K. Yeo, J. Fluid. Mech 196, 359 (1988).

14. P. Carpenter, P. Morris, J. Fluid. Mech 218, 171 (1990).

15. A. Cooper, P. Carpenter, J. Fluid. Mech 350, 231 (1997).

16. P. Drazin, W. Reid, Hydrodynamic Stability (Cambridge University Press, Cambridge, 1981).

17. D. Joseph, Y. Renardy, Fundamentals of Two-Fluid Dynamics (Springer-Verlag, New York, 1993). 